\title{
1 Subcellular localization of mutant P23H rhodopsin in an RFP fusion knockin mouse
}

2 model of retinitis pigmentosa

3

4 Michael A. Robichaux ${ }^{1,3}$, Vy Nguyen ${ }^{1}$, Fung Chan ${ }^{1}$, Lavanya Kailasam ${ }^{1}$, Feng He ${ }^{1}$, John $\mathrm{H}$.

5 Wilson $^{1,2}$, Theodore G. Wensel ${ }^{1}$

6

$7 \quad{ }^{1}$ Verna and Marrs McLean Department of Biochemistry and Molecular Biology, Baylor College

8 of Medicine, 1 Baylor Plaza, Houston, TX, 77030

$9{ }^{2}$ Department of Molecular and Human Genetics, Baylor College of Medicine, 1 Baylor Plaza,

10 Houston, Texas, 77030

$11{ }^{3}$ Departments of Ophthalmology and Biochemistry, West Virginia University, 108 Biomedical

12 Road, Morgantown, WV, 26506

13

14 Short title

15 Subcellular analysis of a $\mathrm{P} 23 \mathrm{H}$ rhodopsin RFP fusion mouse model

16

17 Corresponding author:

18 Theodore G. Wensel, twensel@bcm.edu

19

20 The authors have declared no conflicts of interest. 


\section{Abstract}

The $\mathrm{P} 23 \mathrm{H}$ mutation in rhodopsin (Rho), the visual pigment protein in rod photoreceptor neurons, is the most common genetic cause of autosomal dominant retinitis pigmentosa (adRP), a retinal disease that causes blindness. Despite multiple studies in animal models, the subcellular details of the fate of misfolded mutant Rho in rod photoreceptors have not been completely defined. We generated a new mouse model of adRP, in which the $\mathrm{P} 23 \mathrm{H}-\mathrm{Rh}$ o mutant allele is fused to the fluorescent protein Tag-RFP-T (P23HhRhoRFP). In heterozygotes, outer segments formed, and WT rhodopsin was properly localized there, but mutant P23H-Rho protein was specifically mislocalized in the inner segments of rods. Despite this cellular phenotype, the P23HhRhoRFP heterozygous mice exhibited only slowly progressing retinal degeneration; in ERG recordings, scotopic a-wave amplitudes were reduced by $24 \%$ and $26 \%$ at 30 days and 90 days respectively, and the corresponding scotopic b-waves by $18 \%$ and $24 \%$. Outer nuclear layer thickness was still $80 \%$ of WT at 90 days, but at 364 days had declined to $40 \%$ of WT. Transmission electron microscopy revealed greatly expanded membrane lamellae in the inner segment, and by fluorescence imaging, we determined that the mislocalized P23HhRhoRFP was contained in greatly expanded endoplasmic reticulum (ER) membranes. TUNEL staining revealed a slow pace of cell death involving chromosomal endonucleolytic degradation. Quantification of mRNA for markers of ER stress and the unfolded protein response revealed little or no increases in levels of messages encoding the proteins $\mathrm{BiP}$, CHOP, ATF6, XBP1, PERK, Eif2 $\alpha$ and Derlin-1, but a decreased level of total Rhodopsin (mouse + human) mRNA levels. The decline in the rate of cell death after an initial burst suggests that P23HhRhoRFP mutant rods undergo an adaptative process that prolongs survival despite gross $\mathrm{P} 23 \mathrm{HhRhoRFP}$ protein accumulation in the ER. Because of its slowly progressing nature, and easy visualization of the mutant protein, the P23H-Rho-RFP mouse may represent a useful tool for the future study of the pathology and treatment of $\mathrm{P} 23 \mathrm{H}-\mathrm{Rho}$ and adRP. 


\section{Introduction}

Retinitis pigmentosa (RP) is a hereditary disease of photoreceptor neurons of the retina that causes night blindness, retinal degeneration and, eventually, complete blindness. RP accounts for half of the known cases of all inherited retinal disease (1), affecting 1:4000 in the US (2). Rod and cone photoreceptors, the light-sensing cells in the vertebrate retina are polarized neurons with a specialized outer segment (OS) sensory cilium. The OS is the site of phototransduction, the pathway initiated by the photopigment rhodopsin (Rho) in rods photoreceptors and the cone opsins in cones.

Rho is a prototypical G protein-coupled receptor (GPCR) that is densely packaged as an integral transmembrane protein into the lipid bilayers of membrane discs that fill the rod OS cilium. Each mouse OS contains $\sim 800$ discs, and Rho comprises $>90 \%$ of the total membrane protein content in the OS $(3,4)$. Every day $\sim 10 \%$ of the OS membrane mass in mammalian rods is renewed, as the distal OS discs are shed and engulfed by retina pigment epithelium (RPE) phagocytosis $(5,6)$, and new discs are generated at the base of the OS $(7,8)$. Thus, in each mouse rod photoreceptor $\sim 2.4$ million Rho molecules must be synthesized daily in the inner segment (IS), the biosynthetic compartment of rods, to be delivered to the OS cilium to sustain this renewal (3). This trafficking load - including Rho, other visual proteins, and lipids - must pass through a thin, $300 \mathrm{~nm}$ connecting cilium (CC) bridge located between the IS and OS. The $\mathrm{CC}$ has a cylindrical 9 microtubule doublet cytoskeletal core similar to the structure of the transition zone of other primary cilia (9-11), that extends into the OS and nucleates from a pair of basal body $(\mathrm{BB})$ centrioles located at the distal end of the IS in rods.

The correct localization of Rho throughout the highly specialized compartments of rods is necessary to satisfy the high trafficking rate of Rho molecules in rods, and maintain homeostasis and cell survival (12). Thus, rods are susceptible to genetic mutations to Rho itself, which are the leading cause of autosomal dominant RP (adRP) (13). One point mutation that 
72

73

encodes a proline-to-histidine change at codon $23(\mathrm{P} 23 \mathrm{H})$ in the N-terminus of Rho is the most common single mutation cause of adRP in North America $(14,15)$.

$\mathrm{P} 23 \mathrm{H}-\mathrm{Rho}$ is a misfolding mutation that causes mislocalization of $\mathrm{P} 23 \mathrm{H}-\mathrm{Rho}$ protein in cell culture from the plasma membrane to dense endoplasmic reticulum (ER) cytoplasmic aggregates (16-18). The deleterious effect of the $\mathrm{P} 23 \mathrm{H}-\mathrm{Rh}$ o mutation has been extensively studied in rods in a wide range of animal models, where it causes rod cell death and retinal degeneration with different degrees of severity (e.g., (19-21). In transgenic P23H-Rho frog rods, the mutant Xenopus-P23H-Rho protein is specifically retained and mislocalized in the IS ER (22), while transgenic bovine-P23H-Rho protein in frog rods caused light-induced vesiculation in the IS (23). A zebrafish model expressing mouse-P23H-Rho caused photoreceptor degeneration, abnormal rod OS formation and $\mathrm{P} 23 \mathrm{H}-\mathrm{Rho}$ protein mislocalization throughout the malformed rods in adult fish retina (24).

Transgenic and knockin $\mathrm{P} 23 \mathrm{H}-\mathrm{Rho}$ mouse models have variable retinal degeneration rates that coincide with inconsistent photoreceptor cell phenotypes across models (25-33). Transgenic $\mathrm{P} 23 \mathrm{H}-\mathrm{Rho}$ mice generated in a Rho null background have severely dysmorphic rods, in which mutant $\mathrm{P} 23 \mathrm{H}-\mathrm{Rho}$ is mislocalized in endoplasmic reticulum (ER) surrounding the nucleus (30). In contrast, a knockin mouse model, featuring a mouse-P23H-Rho knockin allele, had dramatic mutant $\mathrm{P} 23 \mathrm{H}-\mathrm{Rh}$ o protein degradation and no detectable ER mislocalization or accumulation phenotype (28). Rod degeneration in the P23H-Rho knockin heterozygotes was fairly rapid (the rod population was reduced by $50 \%$ before P40 (34)) but even more severe in homozygotes. Notably, the undegraded $\mathrm{P} 23 \mathrm{H}-\mathrm{Rho}$ protein in the $\mathrm{P} 23 \mathrm{H}-\mathrm{Rho}$ knockin rods normally localized to the OS and caused abnormal OS disc formation $(28,34)$. One challenge for characterizing the fate of misfolded $\mathrm{P} 23 \mathrm{H}-\mathrm{Rho}$ and testing its effects on different therapeutic approaches, is the difficulty in distinguishing WT rhodopsin from a variant that differs by one amino acid residue. We previously generated a P23H-Rho-GFP fusion knockin mouse to easily visualize the mutant protein in rods, and we observed a gross mislocalization of mutant $\mathrm{P} 23 \mathrm{H}$ - 
Rho-GFP in the IS (35). Furthermore, we found that the mutant fusion protein was largely degraded; however, we did not characterize any subcellular phenotypes in single rods on a nanoscopic level in the P23H-Rho-GFP mouse (21)

For a more in-depth characterization we have generated a new $\mathrm{P} 23 \mathrm{H}-\mathrm{Rho}$ mouse model in order to study mutant $\mathrm{P} 23 \mathrm{H}-\mathrm{Rho}$ protein localization and dynamics, and to serve as a platform for testing gene-based and other therapies. The latter goal is best achieved with a model that has a relatively slow rate of retinal degeneration in line with the slowly progressive course of human vision loss in adRP, which would permit long-term studies. In addition, a relatively non-perturbing tag greatly benefits the testing of therapeutic interventions and strategies.

In this study we introduce a new knockin mouse model of P23H-Rho adRP, in which the mutant P23H-Rho protein is fused to a photostable Tag-RFP-T (36) fusion tag, which we have used to study the mislocalization of the protein. We have also studied the morphological and functional changes that accompany a slowly progressing retinal degeneration

\section{Results}

114 Generation of the P23H-hRho-RFP knockin mouse

We designed a new mouse line in which we introduced into the mouse Rho locus, the human rhodopsin gene (introns and exons) encoding both the $\mathrm{P} 23 \mathrm{H}$ mutation in the first exon

117 and a fusion to Tag-RFP-T at the C-terminus. Tag-RFP-T is a red fluorescent protein (excitation

118 maximum $=555 \mathrm{~nm}$, emission maximum $=584 \mathrm{~nm}$ ) based on a naturally occurring anemone

119 protein engineered for both bright fluorescence and enhanced photostability (36). Notably, Tag-

120 RFP-T fluorescence is compatible with GFP fluorescence for multiplex detection, and it has

121 been used for multiple applications since its design, including as a fluorescent fusion marker 122 (37-39). 
In addition, we added an additional 1D4 signaling sequence to the carboxy-terminus of

124 the P23H-hRho + Tag-RFP-T fusion allele (Fig 1A-B). Our rationale was that the C-terminal

125 RFP fusion tag in the expressed P23H-hRho-Tag-RFP-T (hereafter P23HhRhoRFP) protein

126 may inhibit the endogenous Rho 1D4 sequence leading to artifacts not attributable to the P23H

127 mutation. The additional 1D4 sequence was also shown to be necessary for normal trafficking of

128 transgenic Rho-Dendra fusion protein in Xenopus laevis rods (40).

The P23H-hRho-TagRFP knock-in mice were generated the same way we previously generated P23H-hRho-GFP knock-in mice (35), by gene targeting in the HPRT' embryonic stem

131 (ES) cell line AB2.2 123, which was derived from mouse strain 129SvEv, essentially as

132 described previously $(41,42)$. We introduced the $\mathrm{P} 23 \mathrm{H}$ mutation into the targeting vector by

133 site-directed mutagenesis (QuikChange ${ }^{\circledR}$, Stratagene). An IScel recognition site was

134 engineered into the middle of the first intron in the rhodopsin gene at position 1340 from the

135 start of translation, but it was not used in the experiments described here. The Darwin

136 Transgenic Core Facility, Baylor College of Medicine, electroporated ES cells, selected for

$137 \mathrm{HPRT}^{+} \mathrm{TK}^{-}$cells and injected correctly targeted ES cells into blastocysts from albino C57BL/6-

138 Tyr-Brd mice (43). Founder mice carrying the HPRT-P23H-hRho-TagRFP allele were crossed to

139 GDF-9-iCre mice (44) to remove the HPRT minigene. P23H-hRho-TagRFP (hereafter "P23H-

140 RFP" in reference to the knockin allele) mice were extensively backcrossed to C57BL/6 mice.

141 We validated that the knockin was successful by sequencing genomic DNA from the knockin

142 mouse. We verified expression of the P23HhRhoRFP fusion by fluorescence microscopy of

143 retinas and by immunoblotting (Fig. 1, Fig. 2).

We used $\mathrm{P} 23 \mathrm{H}-\mathrm{RFP} /+$ heterozygous mouse retinas to examine the expression of the

145 mutant fusion protein alongside the WT mouse Rho protein from the wild-type allele (Fig 1B).

146 Both WT mouse Rho protein and the product of the knockin allele were detected in P23H-RFP/+

147 retinal lysates by probing with anti-1D4 antibody. The P23HhRhoRFP fusion protein was 


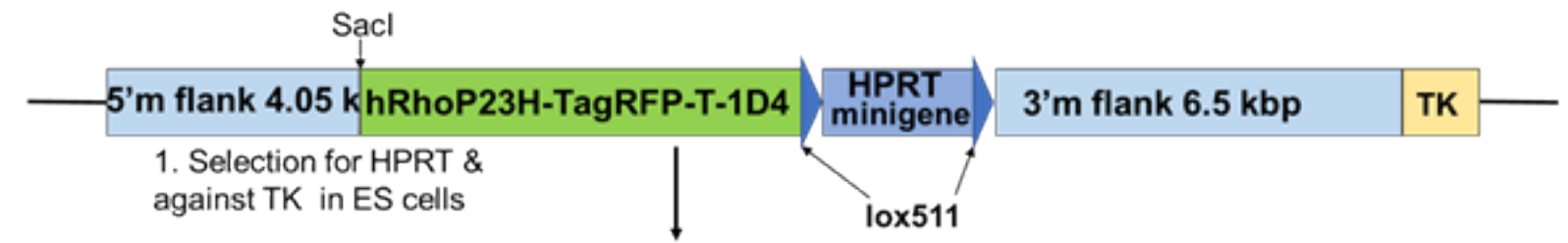

2. Germline transmission through Cre+ oocytes

\section{5' end of mouse gene hRhoP23H-TagRFP-T-1D4}

\section{3' end of mouse gene}

$10 \times 511$

B Human P23H-Rhodopsin-RFP Fusion Gene:
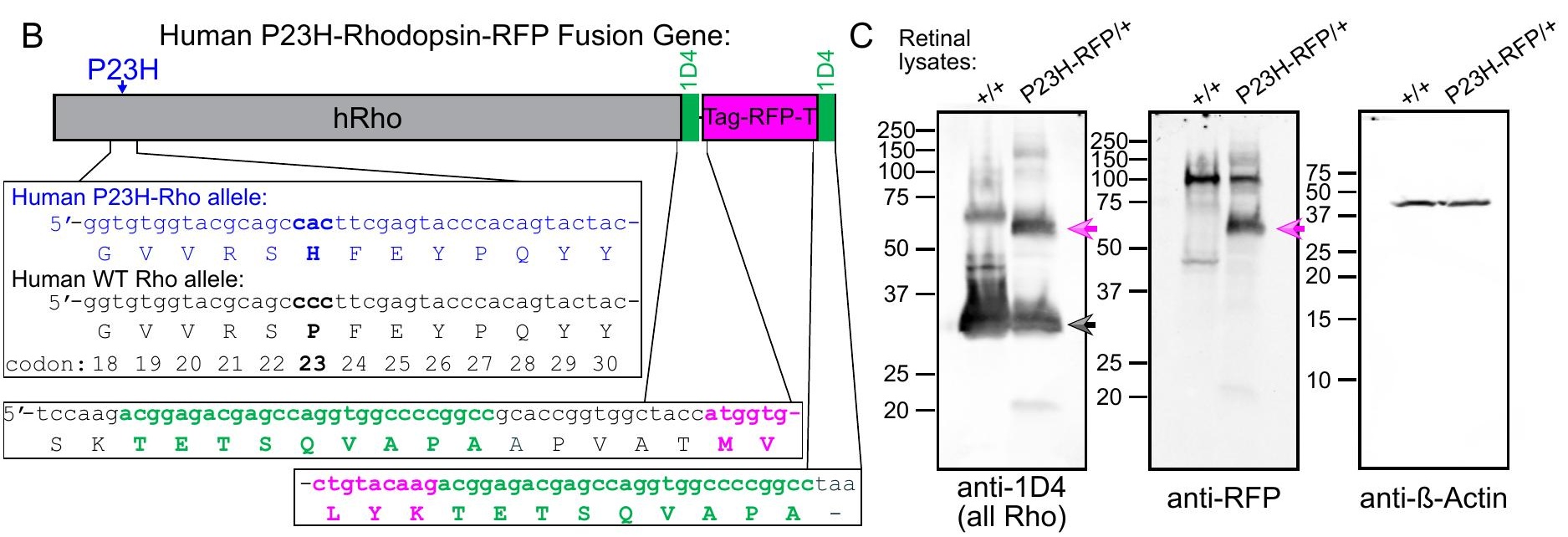

Figure 1. Construction and validation of the P23H-hRho-TagRFP-T knockin mouse. (A) Targeting construct used in embryonic stem cells and resulting gene structure after germline transmission. (B) $A$ map of the knock-in human $\mathrm{P} 23 \mathrm{H}$-rhodopsin-RFP fusion gene. A portion of the sequence of exon 1 from the $\mathrm{P} 23 \mathrm{H}-\mathrm{Rh}$ a allele in the fusion gene (blue) is aligned to the same human wild-type (WT) Rho allele sequence (black). The mutated codon 23 is in bold font. The transition sequence from the 1D4 terminal signal sequence to the Tag-RFP-T with a short linker sequence is shown in the middle sequence panel and the C-terminal sequence with the extra 1D4 epitope sequence appended to the end Tag-RFP-T prior to the stop codon. (C) Western blot confirmation of the P23HhRhoRFP fusion protein expression in $\mathrm{P} 23 \mathrm{H}-\mathrm{RFP} /+$ heterozygous retinas. Retinal lysates are from a wild-type $(+/+)$ mouse, age P22, and a P23H-RFP/+ mouse, age 45; $100 \mu \mathrm{g}$ of total protein from each lysate was loaded onto SDS-PAGE gels. Blot membranes were probed with either of the following antibodies: anti1D4, anti-RFP or anti-beta (ß)-actin (a loading control). Blot scans for the protein ladder were used to mark molecular weight sizes (in kilodaltons, $\mathrm{kDa}$ ) on the left of each blot image. The $\sim 65 \mathrm{kDa}$ $\mathrm{P} 23 \mathrm{HhRhoRFP}$ fusion protein band is present in the P23H-RFP/+ lane in both anti-1D4 and anti-RFP blot scans (magenta arrows). The monomeric mouse Rho protein band is in both lanes in the anti-1D4 blot scan (black arrow). Higher MW species are formed by rhodopsin multimerization. 
detected as a strong $\sim 65 \mathrm{kDa}$ band specifically in P23H-RFP/+ lysates using both anti-1D4 and anti-RFP antibodies, verifying robust expression.

\section{P23HhRhoRFP protein was mislocalized in rod photoreceptor neurons.}

We next tested the fluorescence pattern of the P23HhRhoRFP fusion protein in the retinas of both $\mathrm{P} 23 \mathrm{H}-\mathrm{RFP} /+$ heterozygous and $\mathrm{P} 23 \mathrm{H}-\mathrm{RFP} / \mathrm{P} 23 \mathrm{H}-\mathrm{RFP}$ homozygous mice. The RFP fluorescence is evident in the outer photoreceptor layers of retinal sections from both heterozygotes and homozygotes at age P30 (Fig 2A). In a confocal z-projection of a retinal section from a P30 P23H-RFP/+ heterozygote, $\mathrm{P} 23 \mathrm{HhRhoRFP}$ is most prominently located in brightly fluorescent puncta or "aggregates" within the regions of the inner segments and outer segments of photoreceptors (Fig 2B). Less prominent but visible in this same section is $\mathrm{P} 23 \mathrm{H}$ Rho-RFP fluorescence at the photoreceptor synapses of the outer plexiform layer (OPL) and in the cytoplasm surrounding the photoreceptor nuclei of the outer nuclear layer (ONL) (Fig 2B). Compared to both WT and heterozygotes, the ONL of P30 homozygotes was noticeably thinner based on DAPI+ nuclei staining (Fig 2C). P23HhRhoRFP aggregates were also clearly visible in the ONL of the homozygous retina.

We crossed our new P23H-RFP mouse line with wild-type hRho-GFP mouse lines for dual fluorescent tag multiplex imaging. This approach allowed us to investigate the subcellular dynamics of the mutant P23HhRhoRFP protein relative to hRho-GFP without the $\mathrm{P} 23 \mathrm{H}$ mutation. In addition to the hRho-GFP fusion mouse that we previously reported (41), we also generated a new line with an additional 1D4 signal sequence added to the end of the EGFP sequence ("hRho-GFP-1D4"). As heterozygotes, we could not discriminate any phenotypic difference between these GFP fusion mice.

Both GFP fusion lines were crossed to our new P23H-RFP knockin mouse. In confocal images of retinal sections from an adult hRho-GFP-1D4/P23H-RFP heterozygote we observed a drastic localization difference between hRho-GFP-1D4, which correctly populated the rod 
174 photoreceptor OS cilia, and the P23HhRhoRFP aggregates, which were prominently

175 mislocalized in the rod inner segment layer (Fig 3A). We examined retinal sections from this

176 same hRho-GFP-1D4/P23H-RFP heterozygous mouse line with structured illumination

177 microscopy (SIM) superresolution imaging and observed a clear segregation of P23HhRhoRFP

178 from the hRho-GFP-1D4 in the OS cilia (Fig 3B). Interestingly, in retinal sections from the other

179 heterozygous hRho-GFP/P23H-RFP mice, we found evidence of hRho-GFP mislocalization in

180 the same region as the P23HhRhoRFP aggregates in what appear to be distinct but interwoven

181 membrane compartments (Fig 3C). This result suggests that the added C-terminal 1D4

182 sequence prevents hRho-GFP from being mislocalized with P23HhRhoRFP in the GFP/RFP

183 heterozygous animals.

To visualize the location of the P23HhRhoRFP inner segment aggregates relative to the connecting cilium (CC) and basal body (BB) we used centrin as an antibody marker for the CC and $\mathrm{BB}(45,46)$ in retinal sections from $\mathrm{P} 23 \mathrm{H}-\mathrm{RFP} /+$ mice and imaged by SIM. At age P14, we found many examples of P23HhRhoRFP aggregates that were located just proximal to the BB (Fig 4A). We observed this same sub-BB localization of the RFP aggregates in retinal sections from age P30 P23H-RFP/+ mice (Fig 4B), with some examples of P23HhRhoRFP fluorescence overlapping into the BB region. In retinas from both ages, the RFP fluorescence pattern within these P23HhRhoRFP clumps was discontinuous with dark patches. Morphologically, the P23HhRhoRFP fluorescent aggregates in P14 retina had a compact and defined elliptical shape compared to the aggregates in the P30 retina which appeared more elongated and less structured.

Taken together, fluorescence images of $\mathrm{P} 23 \mathrm{H}-\mathrm{RFP}$ retinas at different ages demonstrate

196 that the P23HhRhoRFP mutant fusion protein was excluded from the OS and mislocalized

197 within aggregates near the BB in the IS and to a lesser degree in the ONL and the

198 photoreceptor synapses. This result suggests that the mutant fusion protein is accumulating in a 


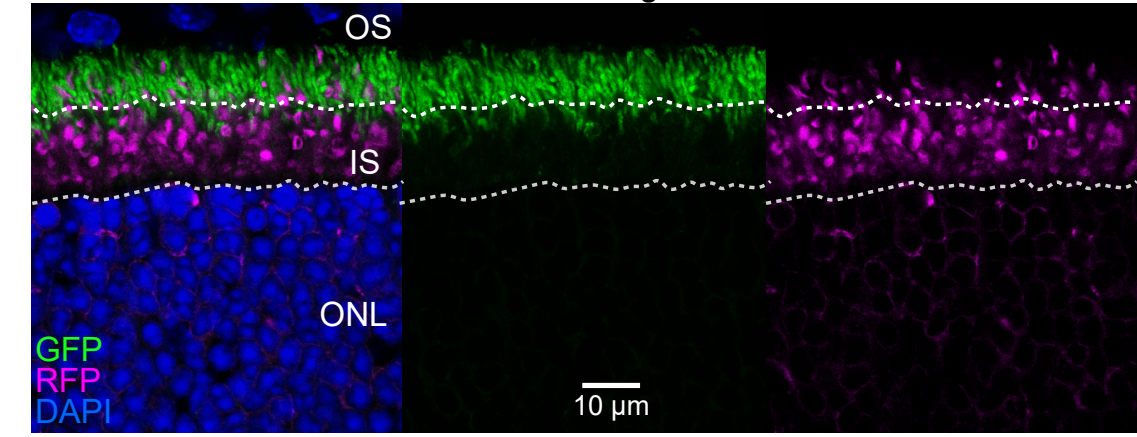

B

mouse: hRho-GFP-1D4/P23H-RFP, age 6 weeks
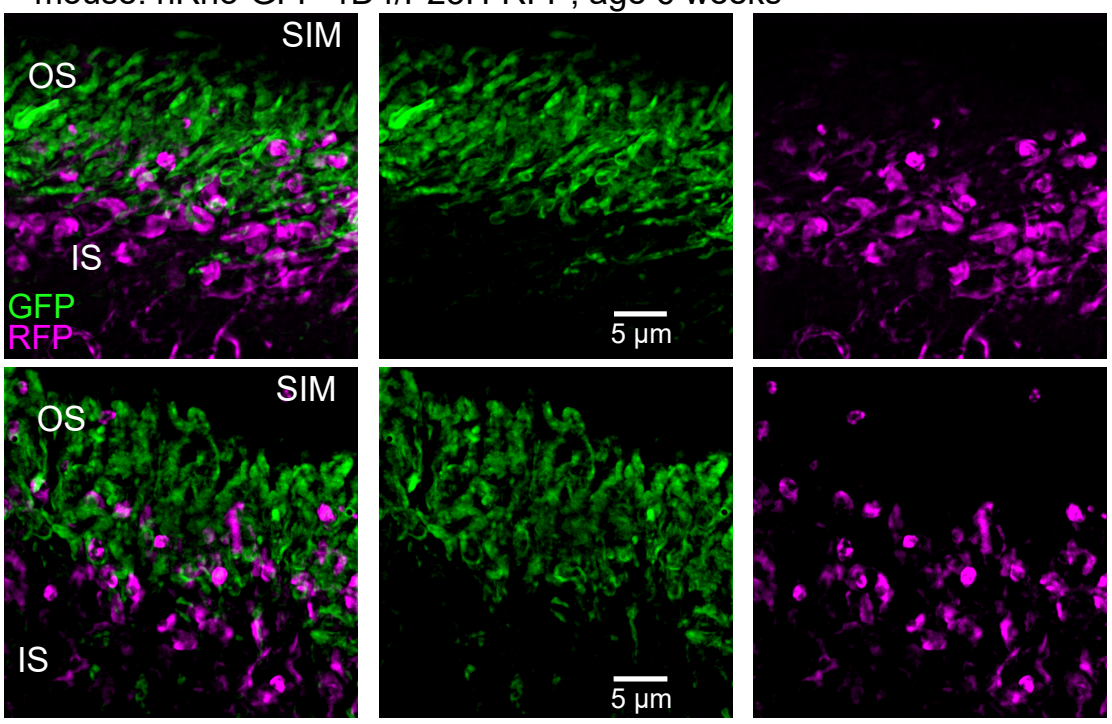

IS

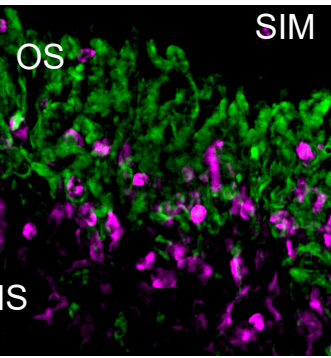

mouse: hRho-GFP/P23H-RFP, age P30
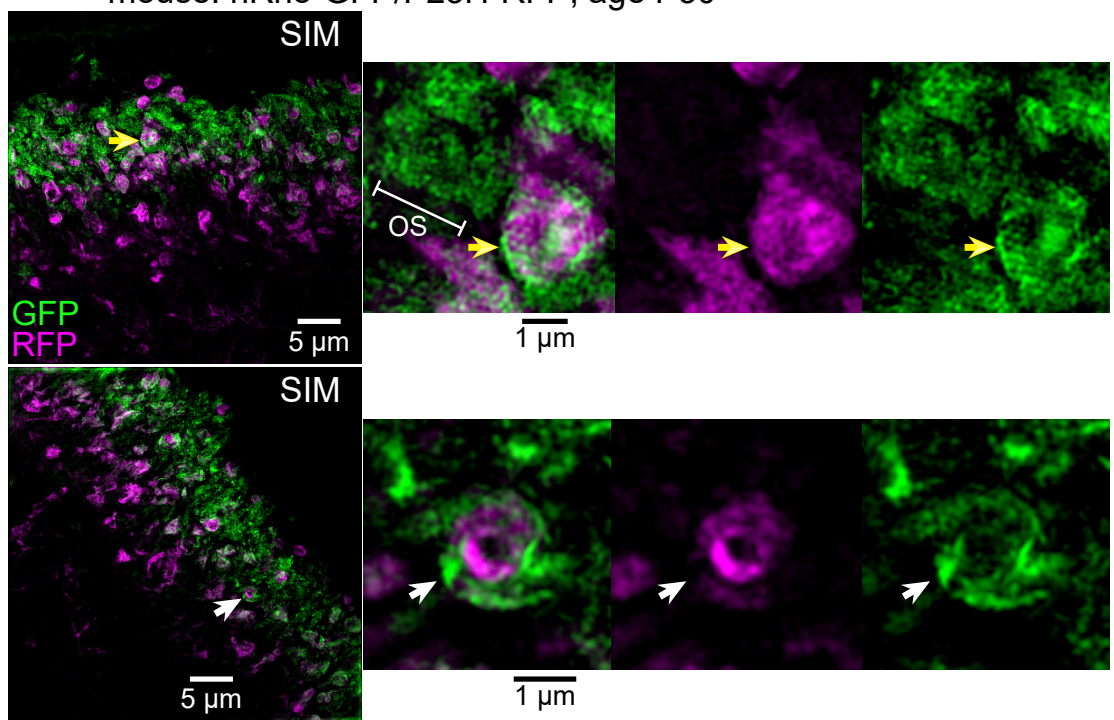

SIM

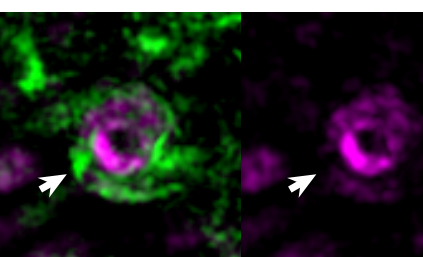

$\overline{1 \mu \mathrm{m}}$
Figure 3. $\mathrm{P} 23 \mathrm{HhRhoRFP}+$ fusion aggregates are localized in the inner segments of rod photoreceptor neurons. (A) In a confocal zprojection image of a retinal section from the hRhoGFP-1D4/P23H-RFP heterozygous mouse retina, at 3 weeks of age, the wild-type hRho-GFP fusion (green), which has an additional C-terminal 1D4 signal sequence, is correctly localized to the outer segments (OS) of rod neurons. In this retina, RFP+ aggregates containing mutant $\mathrm{P} 23 \mathrm{HhRhoRFP}$ protein (magenta) are primarily located in the inner segments (IS) of rods and almost entirely segregated from the GFP+ OS layer. White dotted lines in the figure demarcate the OS/IS boundary and the IS/outer nuclear layer (ONL) boundary. (B) In SIM micrographs of retinal sections from the same heterozygous mouse line at age 6 weeks, GFP+ OS and RFP+ IS aggregates remain segregated with no apparent co-localization. (C) In SIM images of retinal sections from an alternate GFP/RFP heterozygote at age $\mathrm{P} 30$, in which the wild-type hRho-GFP fusion does not have an additional 1D4 signal peptide, the GFP fluorescence is not exclusively located in the OS layer, but rather is partially mislocalized with $\mathrm{P} 23 \mathrm{H}-$ hRho-RFP. In a magnified example, hRho-GFP is colocalized around and within a P23HhRhoRFP aggregate (yellow arrows). In another magnified example, hRho-GFP is wrapped around an P23HhRhoRFP aggregate (white arrow). 
202

203

204

205

206

207

208

209

210

211

212

213

214

215

216

217

218

219

220

221

222

223

224

225

226

227

trafficking stage just prior to the BB and integration into the cilia, and thus it fails to be properly transported to the OS.

$\underline{\mathrm{P} 23 \mathrm{H}-\mathrm{RFP} /+ \text { mice have mild and gradual retinal degeneration. }}$

Next, we tested the effect of the mutant P23HhRhoRFP fusion on retinal health by measuring the rates of retinal degeneration in both $\mathrm{P} 23 \mathrm{H}-\mathrm{RFP} /+$ heterozygous and $\mathrm{P} 23 \mathrm{H}-$ RFP/P23H-RFP homozygous mice. We measured the thickness of the ONL, in which the photoreceptor nuclei were stained for 4',6-diamidino-2-phenylindole (DAPI) fluorescence, in retinal sections from both genotypes and in wild-type $(+/+)$ control retinas from mice at multiple timepoints. Overall, the $\mathrm{P} 23 \mathrm{H}-\mathrm{RFP} /+$ heterozygous retinas degenerated more slowly over time compared to the homozygous retinas as measured by ONL thickness (Fig 5A-B). Our analysis covered the full retina - peripheral to central - to test for any region-specific degeneration caused by the mutant P23HhRhoRFP fusion protein.

At postnatal day 14 (P14) there is no difference in the ONL thickness in the retinas among any of the phenotypes, indicating that the retinas develop normally in the $\mathrm{P} 23 \mathrm{H}-\mathrm{RFP}$ heterozygous and homozygous mutants (Fig 5A-E). Thereafter, the width of the ONL in the heterozygous retinas declined slowly, approaching a final value of $23 \mu \mathrm{m}$ with a time constant of 56 days, while the ONL in the homozygous mutants declined much more rapidly, with a time constant of 12 days. By age P90 the ONL in P23H-RFP/P23H-RFP homozygous retinas was reduced to a single, disorganized layer of nuclei (See Fig 5A, 5E, \& Fig 5G).

Although the ONL thickness in $\mathrm{P} 23 \mathrm{H}-\mathrm{RFP} /+$ heterozygous retinas were significantly reduced in the inferior retina at P60 and P90 (Fig 5B \& 5G), the thickness across all regions of the retina in $\mathrm{P} 23 \mathrm{H}-\mathrm{RFP} /+$ mutants was not significantly different from that of $+/+$ retinas at age P90 (Fig 5G). Notably, compared to P14 P23H-RFP/+ measurements, the P90 P23H-RFP/+ ONL thickness was significantly reduced (Fig 5D). By age P364 it was evident that the ONL had been severely reduced in P23H-RFP heterozygous retinas due to nuclei loss (Fig 5B, see 


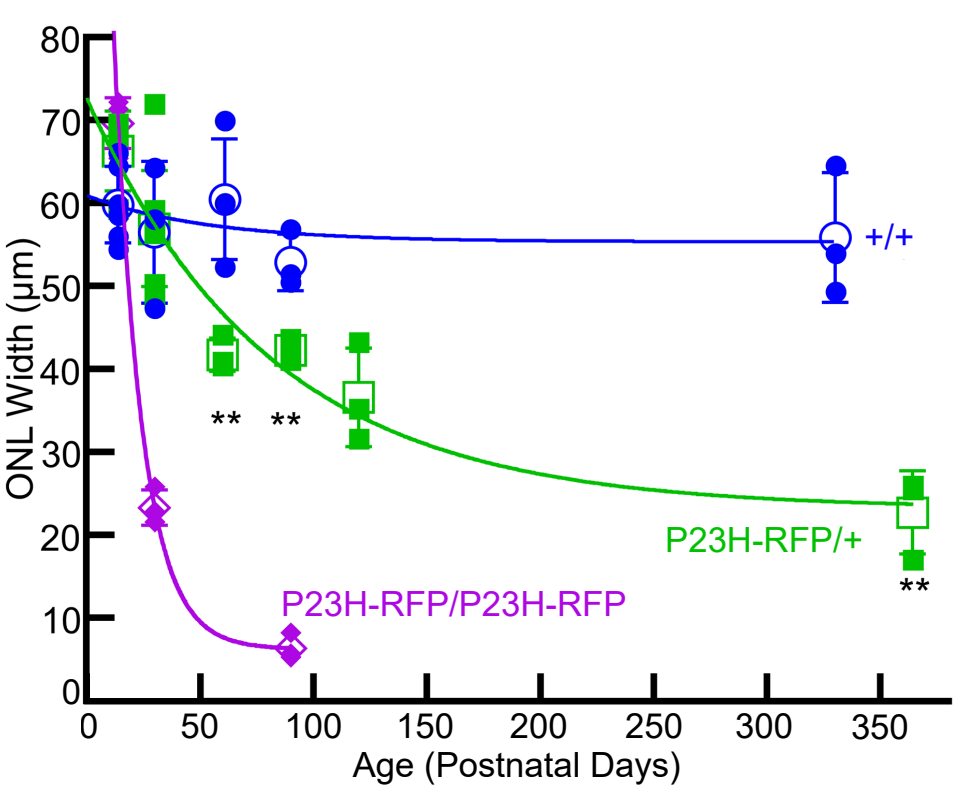

C $+/+$

D

$\mathrm{P} 23 \mathrm{H}-\mathrm{RFP} /+$

E

P23H-RFP/P23H-RFP

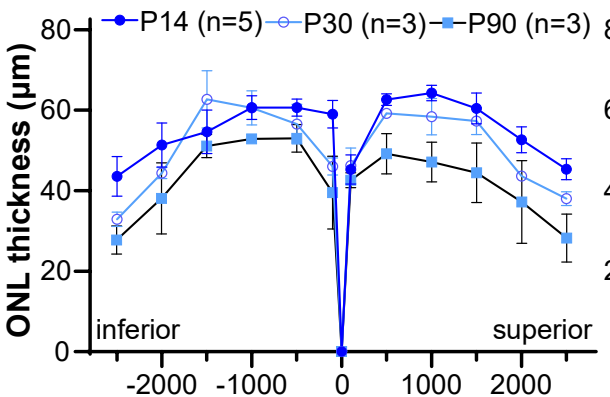

Distance from optic nerve $(\mu \mathrm{m})$

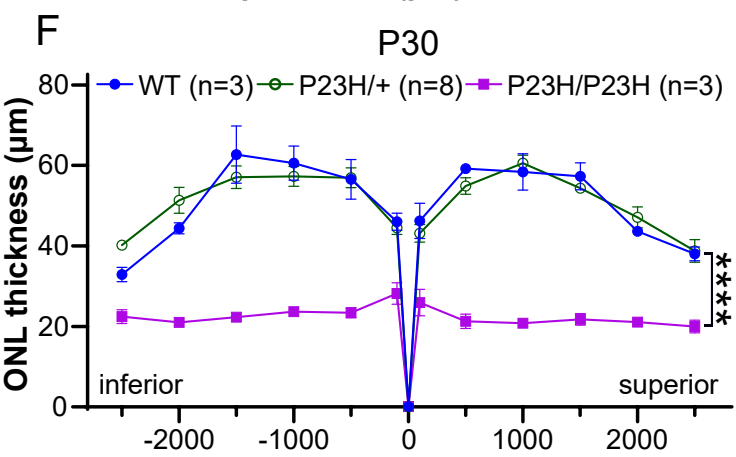

Distance from optic nerve $(\mu \mathrm{m})$

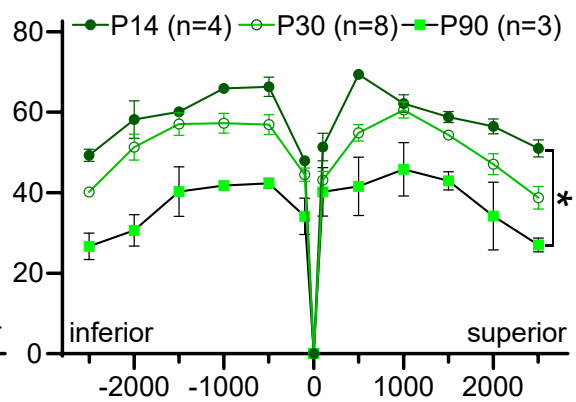

Distance from optic nerve $(\mu \mathrm{m})$

G

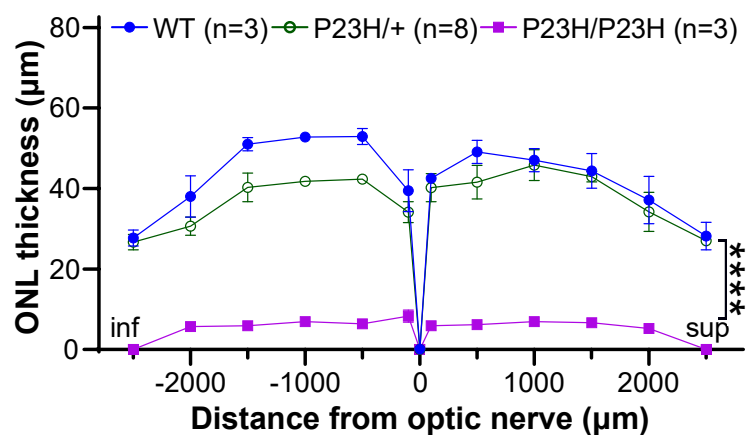


Figure 5. Time course of retinal degeneration in P23H-RFP/+ heterozygous and P23H-RFP/+ homozygous mice. (A) Confocal z-projection images of retinal cryosections from wild-type $(+/+)$, and P23H-RFP heterozygote (het) and P23H-RFP/P23H-RFP homozygous (homo) mice at various time points. DAPI (blue) labels photoreceptor nuclei in the outer nuclear layer (ONL) and the bipolar cell nuclei in the inner nuclear layer (INL). RFP fluorescence (magenta) is primarily located in the inner segment layers of het retinas at all time points, while RFP is also located in the ONL in homo retinas. At P90, the homo ONL is reduced to very few disorganized nuclei surrounded by RFP. (B) Time course plot of ONL thickness between genotypes. Measurements correspond to the ONL thickness of the retina $500 \mu \mathrm{m}$ inferior to the optic nerve. Unfilled shapes correspond to the mean value, and error bars signify standard error of the mean. Solid lines represent fits to exponential decays to plateau, as described in Methods. Unpaired t-tests were calculated to compare $+/+$ and het values for significance. Comparisons with significant differences are: P61 +/+ vs P60 het $\left({ }^{* *} p=0.0081\right)$, P90 +/+ vs P90 het $\left({ }^{* *} p=0.0072\right)$, and P330 +/+ vs P364 het $\left({ }^{* *} p=0.0035\right)$. (C) "Spider" plots of ONL thickness for 13 positions in $+/+$ retinal cryosections from the P14, P30 and P90 time points, spanning positions from the optic nerve to the inferior and superior retina. Positions directly adjacent to the optic nerve position ("0") correspond to $100 \mu \mathrm{m}$ superior and inferior to the optic nerve. The most peripheral positions of each plot correspond to $100 \mu \mathrm{m}$ from the superior and inferior ends of the retina. For each position on the plots, circles correspond to mean values and error bars signify standard error of the mean. (D) Corresponding spider plots for P14, P30, and P90 P23H-RFP/+ het retinal cryosections. Tests for statistical differences between plots from different time points were performed using Two-way ANOVA with Šídák multiple comparisons test. The only test with significance is $\mathrm{P} 14$ het vs $\mathrm{P} 90$ het $\left({ }^{*} \mathrm{P}=0.0215\right)$. (E) Spider plots for P23H-RFP homozygous retinal cryosections at the same time points. Two-way ANOVA with Šídák multiple comparison tests produced significant differences between all time points: P14 vs P30, P14 vs 90 , and P30 vs P90 (all ${ }^{* * *} \mathrm{P}<0.0001$ ). (F) Spider plots for all retinal cryosections at timepoint $P 30$ to compare differences between genotypes. Two-way ANOVA with Šídák multiple comparison tests produced significant differences at P30 between: +/+ vs homo and het vs homo.(both $\left.{ }^{* * * *} P<0.0001\right)$. (G) Spider plots for timepoint P90 to compare genotypes. Two-way ANOVA with Šídák multiple comparison tests produced significant differences at P90 between: +/+ vs homo. and het.vs homo. (both ${ }^{* * *} \mathrm{P}<0.0001$ ). 
example in Fig 5A); the ONL width at 364 days in the heterozygotes was $40 \%$ of that in WT at 360 days.

\section{ERG rod photoreceptor function was moderately diminished in $\mathrm{P} 23 \mathrm{H}-\mathrm{RFP} /+$ mice.}

We next used electroretinogram (ERG) recordings to test and correlate retina visual function to the mild and severe retinal degeneration phenotypes in $\mathrm{P} 23 \mathrm{H}-\mathrm{RFP} /+$ mice and P23H-RFP/P23H-RFP mice, respectively. We found generally that ERG waveforms at age P30 correlated to our retinal degeneration phenotypes between the mutant P23H-RFP genotypes compared to +/+ control mice (Fig 6A). In dark-adapted conditions, the scotopic a-wave amplitudes in P23H-RFP/+ mice were significantly reduced compared to +/+ mice at P30 (Fig $6 \mathrm{~B})$; however, the a-waves stabilized over time and were not further diminished in $\mathrm{P} 23 \mathrm{H}-\mathrm{RFP} /+$ mutants at age P90 compared to P30 (Fig 6C). Scotopic b-wave values were not significantly reduced in P30 P23H-RFP/+ mice compared to +/+ (Fig 6D), and like the a-wave, the b-wave values were not diminished in age P90 P23H/+ mice compared to P30 (Fig 6E). We also measured the implicit times from scotopic ERG recordings, which are times from the a-wave deflection to peak b-wave. In P30 P23H-RFP/+ mice compared to +/+ mice, implicit times were higher at moderate flash intensities, but there was not a significant difference over the entire flash range (Fig 6F).

P23H-RFP/P23H-RFP homozygous mice have essentially no scotopic ERG response (Fig 6A-B and 6D). The minor b-wave response at high flash intensities in homozygotes could be attributed to cones. We also measured the cone ERG response in light-adapted mice from all genotypes at P30. Photopic b-wave amplitudes were recorded in both $\mathrm{P} 23 \mathrm{H}-\mathrm{RFP} /+$ and $\mathrm{P} 23 \mathrm{H}-$ RFP/P23H-RFP mice, and although they appeared slightly lower in amplitude, they were not significantly reduced compared to $+/+$ mice (Fig 6G).

To determine whether there were any visible alterations of cone morphology in the mutants, we used cone arrestin immunofluorescence labeling in our P23H-RFP mice at P30. 
Cones populated the retinas of $\mathrm{P} 23 \mathrm{H}-\mathrm{RFP} /+$ heterozygotes as well as in the retinas of $\mathrm{P} 23 \mathrm{H}-$

257 RFP/P23H-RFP homozygotes despite the massive loss of photoreceptor nuclei in the

258 homozygotes (Fig 7A). At P90 we still observed cones in P23H-RFP/+ heterozygotes, but in

259 P90 homozygotes there were no visible cones remaining, presumably as a result of the nearly

260 complete loss of rods (Fig 7B).

To determine the rate of cell death in our P23H-RFP mice, we used terminal deoxynucleotidyl transferase dUTP nick end labeling (TUNEL) fluorescence. We observed

263 TUNEL+ photoreceptor nuclei in the ONL of retinal sections from both $\mathrm{P} 23 \mathrm{H}-\mathrm{RFP} /+$ and $\mathrm{P} 23 \mathrm{H}-$

264 RFP/P23H-RFP mice (Fig 7C). We measured the number of TUNEL+ nuclei per $\mathrm{mm}^{2}$ area of

265 the ONL at multiple timepoints. At P14, the number of TUNEL+ nuclei in P23H-RFP/+

266 heterozygous retinal sections were comparable to $+/+$ sections, while the rate was significantly

267 greater in homozygous retinal sections at P14 (Fig 7D). By age P30, the number of TUNEL+

268 nuclei was significantly greater in P23H-RFP/+ retina compared to +/+ (Fig 7D); however, at age

269 P90 the TUNEL+ density in heterozygotes was no longer significantly different from that in $+/+$

270 mice (Fig 7D).

271 Overall, we observed that P23H-RFP/+ heterozygous mice have a slow and partial

272 retinal degeneration and mild loss of rod ERG function. At age P90 the heterozygous retinas

273 were still comparable in overall health to control +/+ retinas despite the gross mis-accumulation

274 of P23HhRhoRFP protein in the rod inner segments. The rate of photoreceptor cell death in

$275 \mathrm{P} 23 \mathrm{H}-\mathrm{RFP} /+$ retinas indicates that a moderate burst of degeneration begins after P14, which

276 has slowed by age P90. By comparison, the retinal degeneration and ERG phenotypes in

277 homozygous P23H-RFP/P23H-RFP mice were much more severe, such that at P90, nearly all

278 photoreceptor neurons were lost in the retinas of homozygotes. 
A Age P30: +/+

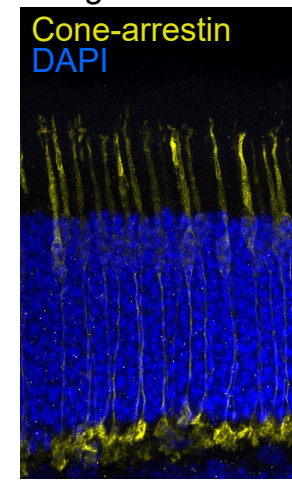

C Age P30: +/+

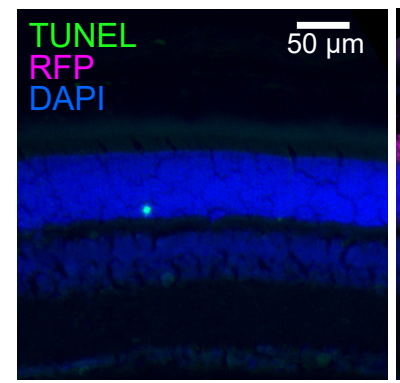

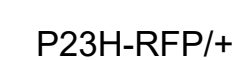
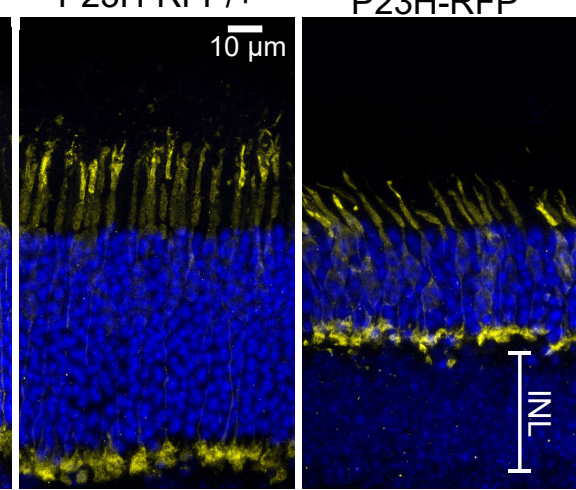

B Age P90: $+/+$

Cone-arrestin DAPI

ope whes and

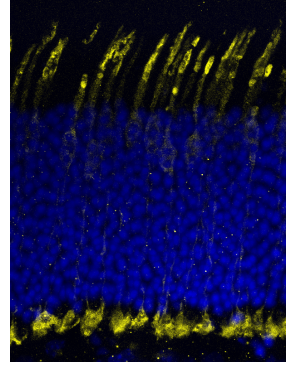

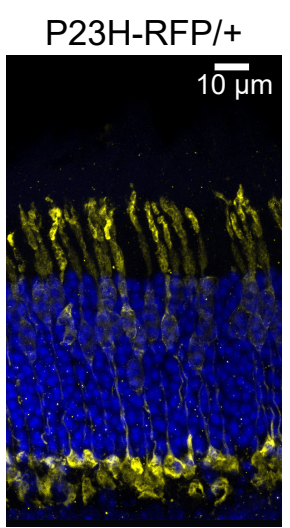

P23H-RFP

$\mathrm{D}$

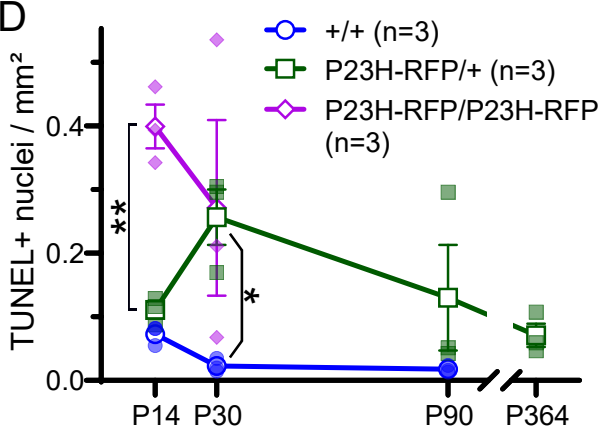

Figure 7. Cone immunolocalization and TUNEL analysis of photoreceptor cell death in P23H-hRho-RFP retinas. (A, B) Examples of cone arrestin immunofluorescence staining (yellow) in retinal sections from ages P30 (A) and P90 (B), among +/+, P23H-RFP/+, and P23HRFP/P23H-RFP mice. DAPI staining (blue) labels both photoreceptor nuclei in the outer nuclear layer (ONL) and in the inner nuclear layer (INL). (C) TUNEL fluorescence analysis of photoreceptor cell in retinal cryosections. Shown are examples of age P30 retinal sections from $+/+, \mathrm{P} 23 \mathrm{H}-\mathrm{RFP} /+$ heterozygous (het), and P23H-RFP/P23H-RFP homozygous (homo) mice with TUNEL + nuclei (green) within the DAPI stained nuclei of the ONL (blue). RFP fluorescence is magenta. (D) Time course plot of aggregate TUNEL+ nuclei $/ \mathrm{mm}^{2}$ measurements among all genotypes at multiple time points. Statistical comparisons among groups were performed using unpaired t-tests. At $\mathrm{P} 14$, homo retinas have statistically more TUNEL+ nuclei compared to both $+/+\left({ }^{*} P=0.0167\right)$ and het $\left({ }^{* *} P=0.0098\right)$ retinas. At $P 30$, het retinas have statistically more TUNEL+ nuclei compared to $+/+$ retinas $\left({ }^{*} \mathrm{P}=0.042\right)$, but the rate of TUNEL+ nuclei between het and $+/+$ retinas is not statistically different at age $P 90(P=0.312)$. 
Quantification of select mRNA levels from $\mathrm{P} 23 \mathrm{H}-\mathrm{RFP} /+$ mouse retinas by $\mathrm{Q}-\mathrm{RTPCR}$.

As ER stress and the Unfolded Protein Response (UPR) have been proposed to be important in neurodegeneration due to misfolded proteins in photoreceptors (47-51), we examined mRNA levels for several markers of these pathways, using two different "housekeeping" genes for normalization, RPL19 and HPRT. Genes whose messages we quantified included those encoding BiP, CHOP, ATF6, Eif2 $\alpha$, PERK, DRL1 and XBP1. None showed a statistically significant increase relative to both "housekeeping" genes (Fig. 8). We also quantified levels of mRNA transcribed from the Rhodopsin locus, using primer pairs that either amplified both human and mouse alleles (mhRho), or the mouse allele only (mRho). Both showed a decrease in message levels in the retinas of heterozygotes relative to those in the response to the presence of aggregated protein.

$\underline{\mathrm{P} 23 \mathrm{H} h \mathrm{Rh} o \mathrm{RFP} \text { protein was mis-accumulated in the inner segment endoplasmic reticulum. }}$ rods, we examined the subcellular structures by transmission electron microscopy (TEM) of ultra-thin retina sections following a tannic acid based staining procedure that densely stains

305 internal membranes (8). At age P14, in P23H-RFP/+ heterozygous rods, we observed distinct

306 membranous accumulations in the IS that matched the shape and morphology of the

307 fluorescent RFP+ aggregates (Fig. 9A-B). Compared to +/+ rods, where cytoplasmic

308 membranes are mostly observed in the proximal IS, the membranous accumulations in heterozygous rods were primarily in the form of semi-organized stacks of folded membranes in 
310 the distal IS. These membranes often filled the mutant IS cytoplasm, apparently distending the

311 width of the IS itself. Indeed, we measured the average maximum IS width of P14 P23H-RFP/+

312 rods to be significantly greater compared to the WT average maximum width (Fig 9, see

313 legend). Despite these large aberrant IS membranes in $\mathrm{P} 23 \mathrm{H}-\mathrm{RFP} /+$ rods, the morphologies of

314 the BB, CC and OS discs were relatively normal and indistinguishable between P23H-RFP/+ 


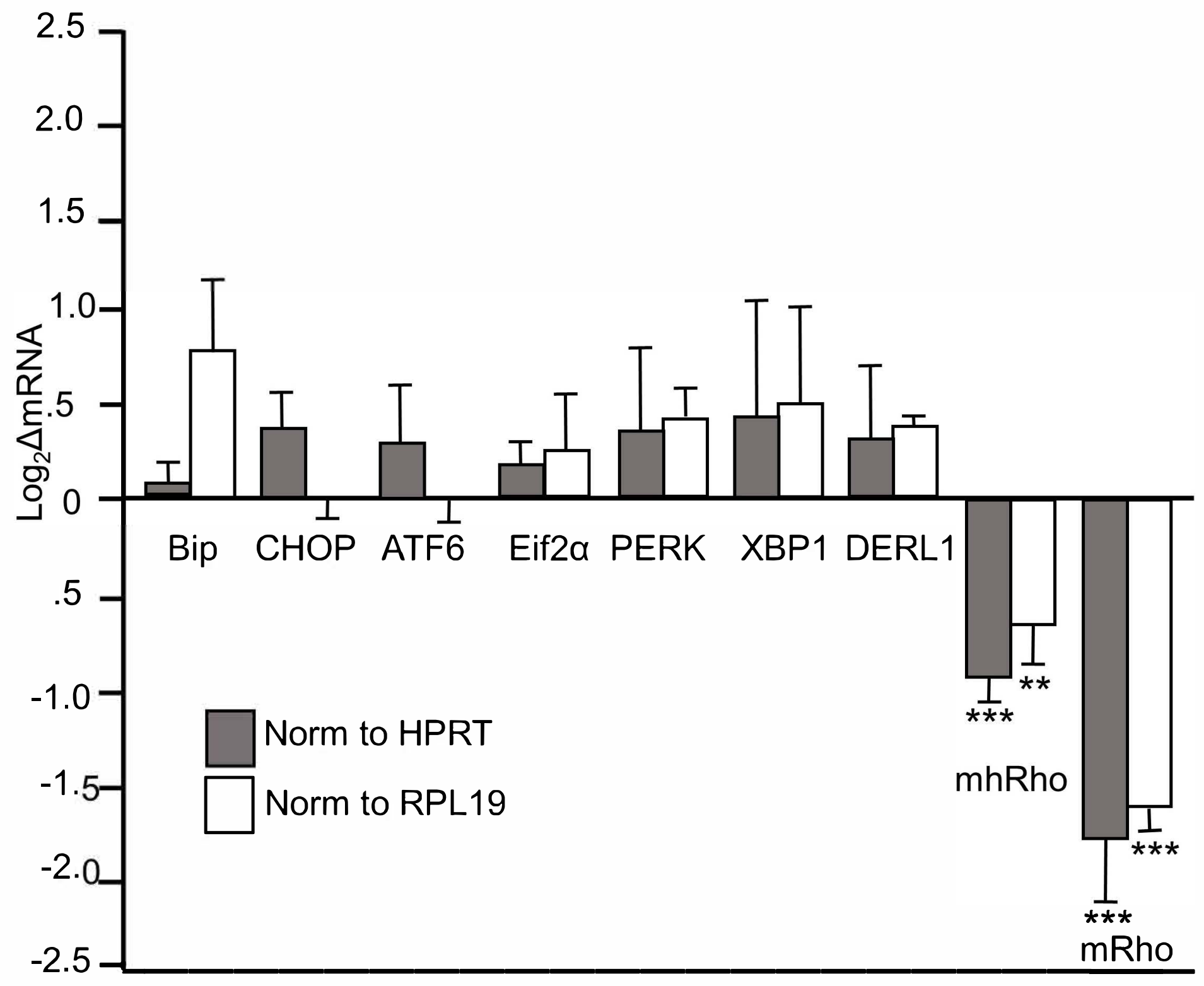

Figure 8. mRNA levels of ER stress and Unfolded Protein Response markers are near normal in P23H-RFP/+ heterozygous retinas at postnatal day 30 . Results of Q-RTPCR measurements of the indicated messages in RNA extracted from retinas of heterozygotes as compared to wild-type $(+/+)(n=3$ for both), normalized to HPRT (dark grey bars) or RPL19 (open bars). Statistical comparisons were made with two-tailed t-tests.: mhRho vs. RPL19 ${ }^{* *} \mathrm{P}=0.0099 ; \mathrm{mhRho}$ vs. HPRT ${ }^{* * *} \mathrm{P}=0.000741$.; $\mathrm{mRho}$ vs. RPL19 ${ }^{* * *} \mathrm{P}=0.000585$. mRho vs. HPRT ${ }^{* * *} \mathrm{P}=0.000542$. 
and WT rods at P14, except for the length of the CC, which was significantly longer in mutant rods (Fig 9A-B, see legend).

We also used TEM to examine the morphology of the RFP+ IS aggregates in P23H-

RFP/+ rods at P30, where the RFP granule fluorescence appeared less organized than in P14 rods (Fig 4A-B). With TEM, in age P30 P23H-RFP/+ rods, we also observed accumulated IS membranes; however, they did indeed appear more dysmorphic than those at P14, with

324 examples of the membranes wrapping around themselves in whorls (Fig 9C, magenta arrows).

325 As in P14 P23H-RFP/+ retinal sections, the aberrant rod IS membranes at P30 were located

326 among normally formed OS disc stacks, and the CC and BB structures were also

327 morphologically normal at P30 (Fig 9D). Unlike P14 rods, however, we observed some defects

328 in OS membrane morphology at the base of the OS in some P30 P23H-RFP/+ rods. These

329 defects included vesicular and misshapen discs, and an unbound, splayed OS axoneme (Fig

330 9C-D, green arrows). The results indicate that the mutant P23H-Rho-RFP leads to massive

331 alterations of inner segment membrane structure, accompanied by a least partial disruption of

332 outer segment morphology.

To determine the nature of the membranes in which the RFP fusion protein is located,

334 we used immunofluorescence with antibodies for the endoplasmic reticulum (ER) antigens

335 BiP/GRP78 (binding immunoglobulin protein/glucose-regulated protein 78, an ER lumen

336 chaperone protein) and KDEL (an ER-specific tetrapeptide folding tag), and for the Golgi

337 antigen GM130 (a Golgi-specific membrane marker). In confocal z-projections, we found that

338 BiP ER immunolabeling was co-localized with the RFP+ IS aggregates in rod cells from P14

$339 \mathrm{P} 23 \mathrm{H}-\mathrm{RFP} /+$ retinal sections (Fig 10A). Compared to +/+ BiP labeling, which was largely

340 located in the proximal IS, BiP staining in the mutant $\mathrm{P} 23 \mathrm{H}-\mathrm{RFP} /+$ retina was extended into the

341 distal IS and colocalized with the RFP+ aggregates. This result demonstrates that the

342 membrane stacks we observed in the IS of mutant rod via TEM are greatly expanded ER

343 membranes. The Golgi 
bioRxiv preprint doi: https://doi.org/10.1101/2021.10.11.463949; this version posted October 11, 2021. The copyright holder for this preprint

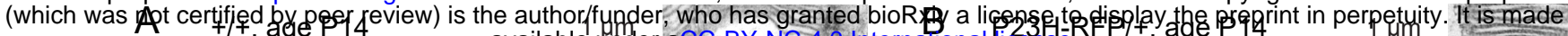
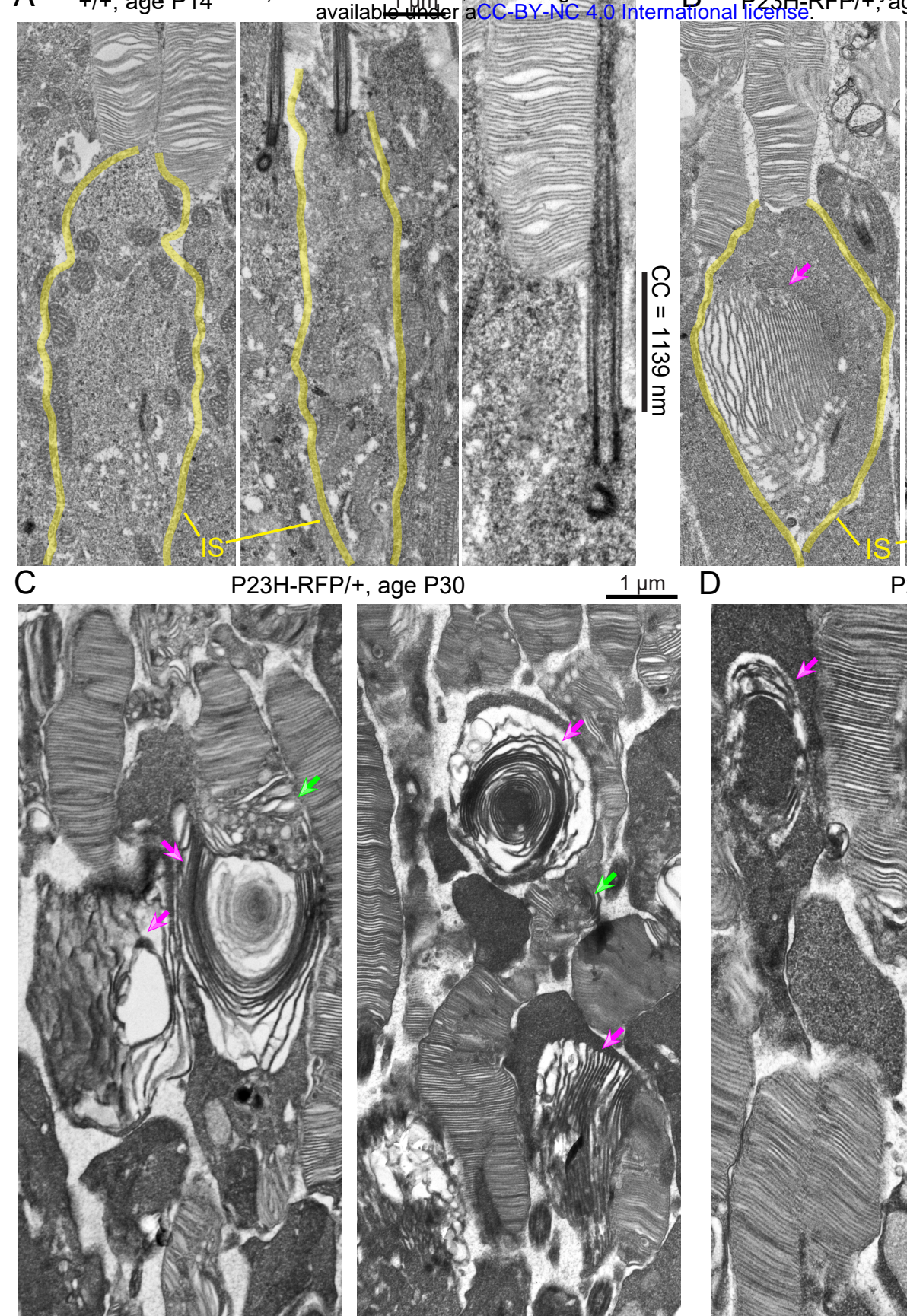

++ , age P30

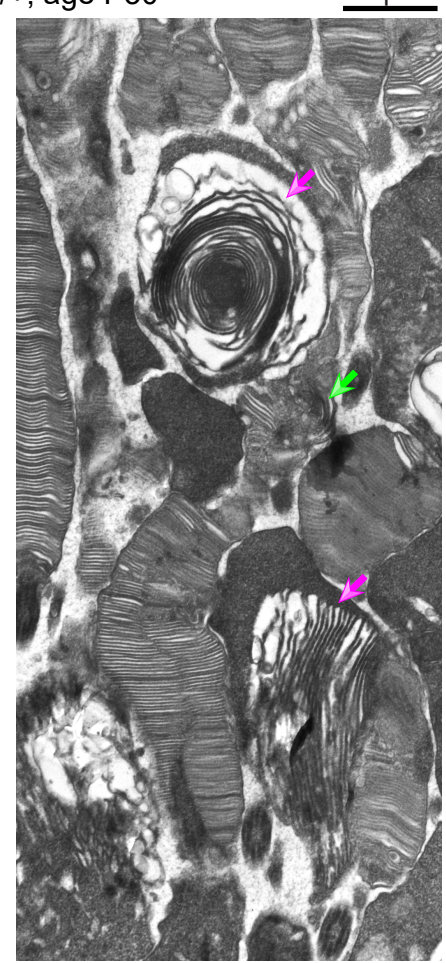

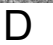

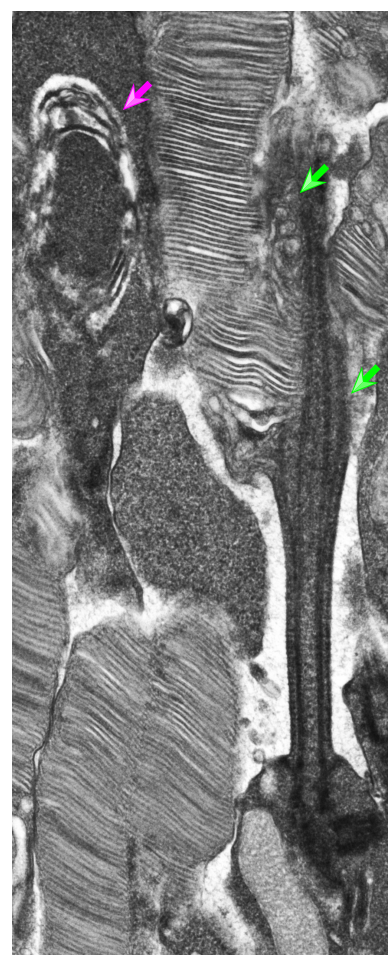

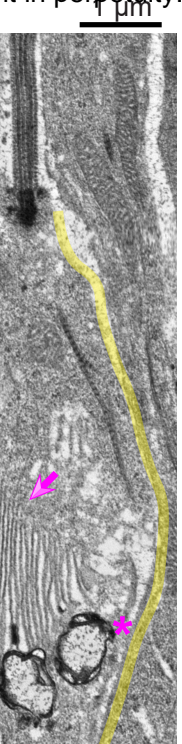

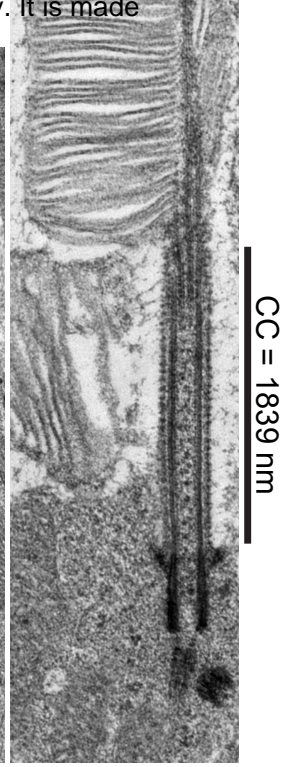

/+, age $P 30$

$500 \mathrm{~nm}$

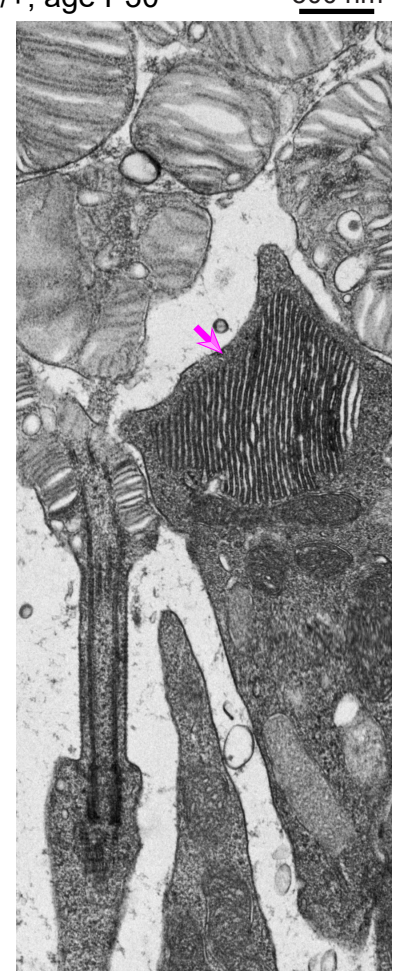

Figure 9. P23H-Rho-RFP/+ mutant rod photoreceptor neurons have distended inner segments filled with ectopic membranes. (A-B) Conventional transmission electron microscopy (TEM) images of rod photoreceptor neurons at age P14 from either (A) $+/+$ or (B) P23H-RFP/+ heterozygous (het) mice. In each example rod, the inner segment (IS) is outlined with yellow lines. The IS max width in P14 $\mathrm{P} 23 \mathrm{H}-\mathrm{RFP} /+$ rods is significantly greater than $+/+$ rods (het: $2.967 \mu \mathrm{m} \pm 0.508 \mu \mathrm{m}$ (standard deviation, sd) $(n=14)$ vs $+/+: 1.974 \pm 0.481 \mu \mathrm{m}(\mathrm{sd})(\mathrm{n}=19), P<0.0001$, unpaired t-test). Ectopic stacks of IS membranes in the swollen $\mathrm{P} 23 \mathrm{H}-\mathrm{RFP} /+$ IS are marked with magenta arrows. Double-membraned autophagy compartments are marked with magenta asterisks in the mutant P23H-RFP/+ IS. In magnified views of example connecting cilia (CC) from each genotype, the length of the $\mathrm{CC}$ - measured by the densely stained CC membrane - is indicated. In aggregate, the length of the CC in P23H-RFP/+ rods is significantly greater than $+/+C C$ (het: $1.548 \mu \mathrm{m} \pm 0.206 \mu \mathrm{m}(\mathrm{sd})(\mathrm{n}=13) \mathrm{vs}+/+: 1.27 \mu \mathrm{m} \pm 0.247$ $\mu \mathrm{m}(\mathrm{sd})(\mathrm{n}=11), \mathrm{P}=0.0066$, unpaired t-test). (C) At age $\mathrm{P} 30$, the ectopic IS membranes in $\mathrm{P} 23 \mathrm{H} /+$ rods appear more dysmorphic compared to P14 (magenta arrows). In addition, some outer segments disc membranes within or adjacent to $\mathrm{P} 23 \mathrm{H}-\mathrm{RFP} /+$ rods with IS defects are also disrupted and appear dysmorphic (green arrows). (D) In examples of the CC and basal OS regions of P30 P23H/+ rods, the structure of the CC and basal body remain intact despite being adjacent to ectopic IS membranes (magenta arrows); however, there is evidence that basal OS disc morphogenesis is disrupted possibly due to OS axoneme instability (green arrows). 
network was unaffected in P14 P23H-RFP/+ retinas and was not co-localized with RFP+ aggregates (Fig 10B).

We used SIM superresolution microscopy to examine the morphology of the ER and Golgi more closely in individual rods in $\mathrm{P} 23 \mathrm{H}-\mathrm{RFP} /+$ heterozygous and $+/+$ retinas at $\mathrm{P} 30$. We added centrin immunolabeling to label the $\mathrm{CC}$ and $\mathrm{BB}$ in these SIM experiments. In P30 P23HRFP/+ retinas, we again observed BiP co-localization with RFP aggregates in the IS (Fig 10 B$\mathrm{C}$, white arrows). The BiP+ ER lumen surrounded and was intercalated with the mislocalized P23HhRhoRFP protein that appeared aggregated within the ER membranes. We also observed $\mathrm{BiP}+\mathrm{ER}$ in other regions of the IS including at the BB (Fig $10 \mathrm{~B}-\mathrm{C}$, yellow arrows). In control wild-type (+/+) P30 retinas, the BiP-positive ER is located throughout the IS in a reticulated morphology that extends to the BB as well (Fig 10E). We observed similar KDEL+ ER localization in P30 P23H/+ rods: both co-localized within RFP+ aggregates (Fig 10F, white arrows) and in the BB region (Fig 10F, yellow arrows); however, KDEL labeling was more punctate than BiP labeling. In P30 +/+ rods, KDEL was also localized throughout the IS and in the BB region (Fig 10G). Although there is much evidence of co-localization of RFP and ER marker signal, the ER markers are not uniformly distributed throughout the clumps of RFP signal, and there are large sections of RFP-positive aggregates without ER marker signal. We also used SIM to examine the morphology of GM130+ Golgi in P30 P23H-RFP/+ rods. As before we observed the Golgi in the proximal IS of P23H-RFP/+ retinas and segregated from the RFP+ aggregates and the centrin+ CC/BB. In some mutant heterozygous rod IS, however, we observed smaller GM130+ Golgi membranes within the RFP+ aggregates

369 (Fig 10H, white arrows). Also, we found some examples of P30 P23H-RFP/+ rods with more 370 elaborate Golgi that extended into the distal IS and nearby RFP+ aggregates (Fig 10H, yellow 371 arrows). In SIM images of P30+/+ retina immunolabeled for GM130 and centrin, we found most 372 of the Golgi in the proximal IS and dissociated from the centrin+ cilium. Interestingly, however, 


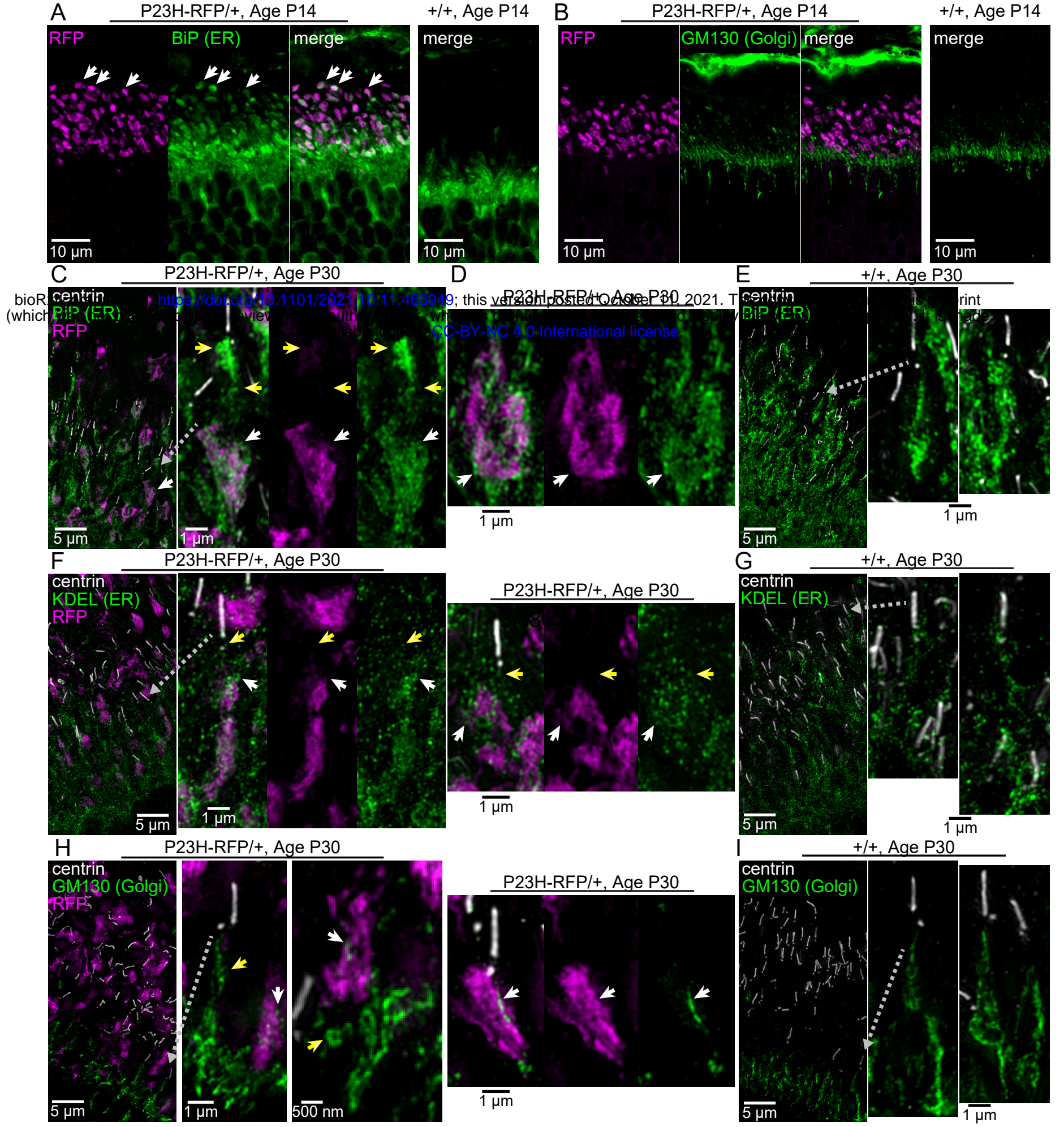

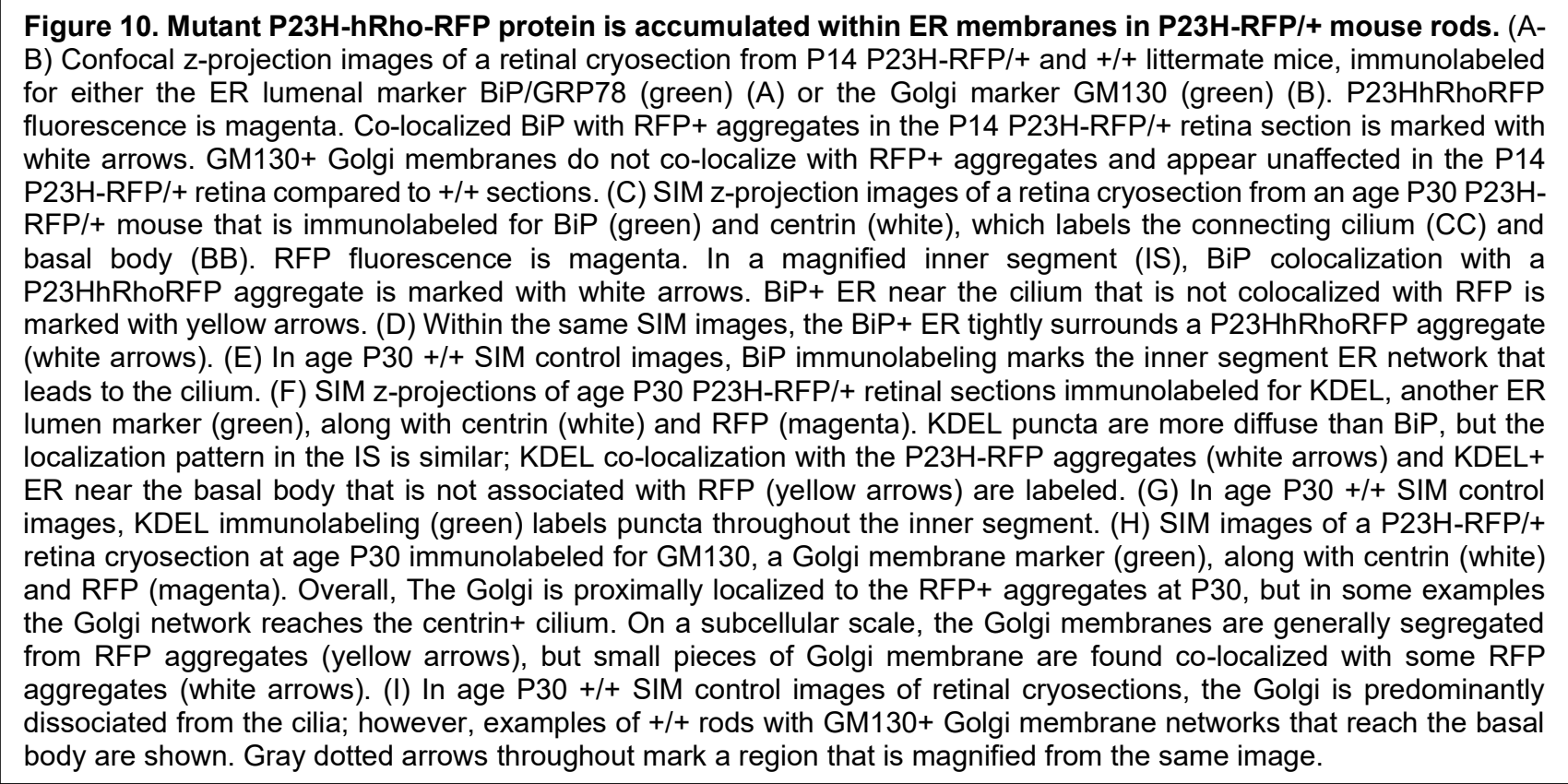


bioRxiv preprint doi: https://doi.org/10.1101/2021.1011.463949; this version posted October 11,2021 . The copyright holder for this preprint

(which was not certified by peer review) is the author/funder, who has granted bioRxiv a license to display the preprint in perpetuity. It is made available under aCC-BY-NC 4.0 International license.

377 we did observe some examples of $+/+$ rods in which the Golgi network was more elaborate and

378 extended up to the BB (Fig 10I).

379 Finally, in our SIM images we observed both $\mathrm{BiP}+$ and $\mathrm{KDEL}+\mathrm{ER}$ localized with the

380 mislocalized P23HhRhoRFP protein in the region of rod synapses in the OPL (outer plexiform

381 layer) of P30 P23H-RFP/+ retinas (Fig 11A-B). GM130-positive Golgi was not localized in the

382 OPL of P30 P23H-RFP/+ retinas (Fig 11C). 


\section{Discussion}

The P23HhRhoRFP mouse we introduce here is a potentially useful animal model for adRP that can reveal the subcellular and molecular pathology of the misfolding $\mathrm{P} 23 \mathrm{H}-\mathrm{Rho}$ mutation on the long-term health of mammalian rod neurons. Tag-RFP-T fluorescence in these mice enables both gross and nanoscopic analysis of $\mathrm{P} 23 \mathrm{H}-\mathrm{Rh}$. ER accumulation in vivo. The WT-hRho-GFP/P23HhRhoRFP dual color heterozygote demonstrates the different ways the cell processes these two proteins with similar fusions, but with and without the $\mathrm{P} 23 \mathrm{H}$ rhodopsin mutation (Fig 3). WT-Rho-GFP fusion protein is restricted almost exclusively to the OS in rods of the Rho-GFP heterozygotes (52), whereas the same fusion construct with a P23H mutation was largely confined to the inner segment and ONL (35), as observed in the new model reported here, consistent with the notion that the $\mathrm{P} 23 \mathrm{H}$ mutation is responsible for the disruption in normal trafficking, likely as a result of misfolding (18). Furthermore, with superresolution microscopy, we observed P23HhRhoRFP localization proximal to the connecting cilium and basal body in P23H-RFP/+ heterozygous rods and completely excluded from the OS (Figs 2-3).

401 The exclusive IS mislocalization of the P23H-Rho-RFP protein is unlike P23H-Rho localization 402 in other mouse models, in which there is some detectable OS transport despite other mislocalization and degradation phenotypes $(25,27,31,34)$. $\mathrm{P} 23 \mathrm{H}-\mathrm{Rho}$ to the IS, rod neuron degeneration in the homozygotes was more severe. In the 406 heterozygotes, despite dramatic $\mathrm{P} 23 \mathrm{H}-\mathrm{Rho}$ mislocalization and a burst of rod cell death around 407 age $\mathrm{P} 30$, the progression of degeneration in $\mathrm{P} 23 \mathrm{H}-\mathrm{RFP} /+$ retinas was only partial and relatively 408 slow. By comparison, in the "untagged" P23H-Rho knockin mouse line, heterozygotes lose $43 \%$ 409 of their rod population relative to WT by P63 (28); we do not observe such a severe reduction 410 until age P120 (Fig 5B). 
The stability of the P23H-RFP/+ heterozygous rods over time enables long term studies

412 in these mice and suggests an adaptation in these rods that provides a neuroprotective effect

413 despite the ER accumulation of $\mathrm{P} 23 \mathrm{H}-\mathrm{Rho}$ protein. This effect may be related to an ER stress

414 adaptation like the unfolded protein response (UPR), which was originally characterized based

415 on an increase in BiP/GrP78 and C/EBO protein (Chop) mRNA levels - indicators of an

416 activation of the pancreatic endoplasmic reticulum kinase-like endoplasmic reticulum kinase

417 (PERK) UPR pathway - in transgenic P23H-Rho rats (53). Also in P23H-Rho transgenic rats,

overexpression of BiP/Grp78 preserved ERG rod function (54), and overexpression of the BiP-

binding ER chaperone ERdj5 preserved photoreceptor survival (55). Although we found no co-localized with the ER-accumulated P23H-Rho-RFP protein (Fig. 9). we observed in P23H-RFP/+ rods with TEM (Fig 8) could a be compensatory ER stress

432 mechanism in response to an overload of ERAD and proteasome degradation. Such an 433 adaptation warrants further study. In TEM images of P14 P23H-RFP/+ rods, we also observed 434 double membrane autophagosome-like structures adjacent to the ER (Fig 8A), indicating an 435 autophagy component to the P23H-RFP pathology. Similar double membrane vesiculations 436 were observed in transgenic bovine-P23H-Rho Xenopus tadpoles that were exposed to light 
437 (23), and more recently LC3-positive autophagosomes were localized adjacent to P23H-Rho 438 protein in the inner segments of these same Xenopus rods expressing bovine-P23H-Rho (58).

We observed a deterioration in the morphology of the accumulated ER IS membranes in P23H-RFP/+ rods from age P14 to P30 with both RFP fluorescence (Fig 4) and TEM (Fig 8). In addition to large IS membrane whorls in the IS at P30 (Fig 8C), the ER membrane structures were more disorganized than the tight membrane stacks at P14. We also observed ER at the 443 photoreceptor synapse carrying mislocalized P23HhRhoRFP protein in the OPL of P30 P23H-

444 RFP/+ retinas (Fig 10). Rhodopsin mislocalization to the synapse layer and in the ONL cytoplasm was previously observed in $\mathrm{P} 23 \mathrm{H}-\mathrm{Rho}$ transgenic mice (31). This suggests that the ER expands throughout the entire cytoplasmic space in rods by P30 as a broad response to the accumulation of misfolded $\mathrm{P} 23 \mathrm{H}-\mathrm{Rho}$ protein in the ER. morphology of the $\mathrm{CC}$ and the OS disc structure was normal in $\mathrm{P} 23 \mathrm{H}-\mathrm{RFP} /+$ rods at age $\mathrm{P} 14$ (Fig 8A-B). At P30, the OS discs is some P23H-RFP/+ rods were slightly dysmorphic, but the 451 CC structure appeared structurally intact, albeit longer than in +/+ rods (Fig 8C-D). The CC 452 elongation $\mathrm{P} 23 \mathrm{H}-\mathrm{RFP} /+$ rods is a surprising result. Such an elongation phenotype was also 453 described in knockout mouse models for 2 CC-localized proteins: male germ cell-associated 454 kinase (Mak) and Huntingtin (59, 60). In both knockouts, the elongated CC was accompanied 455 with aberrant OS morphology and Rho mislocalization. One possible cause of the elongated CC 456 in $\mathrm{P} 23 \mathrm{H}-\mathrm{RFP} /+$ mutant rods may be an early imbalance of Rho expression during an early 457 ciliogenesis stage due to mutant P23H-Rho-RFP expression.

$459 \mathrm{P} 23 \mathrm{H}-\mathrm{RFP}$ mouse retinas is additional evidence that $\mathrm{P} 23 \mathrm{H}-\mathrm{RFP} /+$ mutant rods undergo a long460 term neuroprotective response. Unlike in P23H-RFP/P23H-RFP homozygous retinas where the 461 TUNEL + rate in the ONL is elevated at P14, the rate of TUNEL+ nuclei spikes at P30 in P23H/+ 462 heterozygous retinas and returns to a level not significantly higher than +/+ retinas by P90 (Fig. 
7C). Interestingly, a similar spike in TUNEL staining was also observed in both $\mathrm{P} 23 \mathrm{H}-\mathrm{Rho}$

464 transgenic rats at age P18 vs age P30 (32) and in the untagged P23H-Rho knockin mouse at 465 age P19 vs P31 (61).

In conclusion, our RFP fusion knockin model of adRP retinal degeneration caused by the

$467 \mathrm{P} 23 \mathrm{H}$ rhodopsin mutation is a unique model for the disease with clear phenotypes that are

468 traceable with both fluorescence microscopy and TEM. The mutant rods in our P23H-RFP/+

469 mice demonstrate an adaptation that promotes rod photoreceptor survival, and thus the

470 heterozygous mutant retinas have a mild rate of retinal degeneration. As such, our model will be

471 useful to test the variety of proof-of-concept adRP therapies that have been developed across

472 all $\mathrm{P} 23 \mathrm{H}-\mathrm{Rho}$ models in the field. These include: genetic suppression and replacement

473 strategies $(62,63)$, CRISPR/Cas9 mutant allele deletions (64-66), genetic inhibition of the

474 autophagy-activating $\operatorname{Atg} 5$ (67), caspase pathway inhibitors $(61,68)$, a small molecule inhibitor

475 of the photoreceptor specific transcriptional modulator Nr2e3 (69), and pharmacological

476 treatments with valproic acid and other histone deacetylase (HDAC) inhibitors (70). Future

477 studies that investigate the mechanisms of action for these therapies can be tested in this

$478 \mathrm{P} 23 \mathrm{H}-\mathrm{RFP}$ mouse for the development of the most efficient and synergistic treatments of $\mathrm{P} 23 \mathrm{H}-$

479 Rho and adRP.

480

481

\section{Materials and Methods}

482 Animals

The P23H-hRho-TagRFP knock-in mice were generated the same way we previously

484 generated P23H-hRho-GFP knock-in mice (35), by gene targeting in the HPRT'- embryonic stem

485 (ES) cell line AB2.2 123, which was derived from mouse strain 129SvEv, essentially as described previously $(41,42)$. The targeting plasmid plasmid contained 5 ' and 3' sequences identical to flanking sequences of the mouse rhodopsin gene, an intervening sequence corresponding to the human Rhodopsin gene encoding the $\mathrm{P} 23 \mathrm{H}$ mutation found in patients, a 
C-terminal fusion with the fluorescent protein Tag RFP-T, a final C-terminal peptide corresponding to the C-terminal 9 residues of human rhodopsin (TETSQVAPA, the epitope for

491 the 1D4 monoclonal antibody and a putative outer segment targeting signal), a STOP codon, 492 and an endogenous polyadenylation signal, followed by an expression cassette (minigene) for 493 human hypoxanthinephosphoribosyltransferase (HPRT). The HPRT minigene was flanked by 494 loxP sites, so it could be looped out in vivo by passing through the germline of GDF-9-iCre mice 495 (44) expressing Cre recombinase in oocytes. The plasmid also contained a minigene for 496 Herpes Simplex Virus thymidine kinase (TK) outside the region of homology for negative selection against non-homologous insertion. We introduced the $\mathrm{P} 23 \mathrm{H}$ mutation into the targeting vector by site-directed mutagenesis (QuikChange ${ }^{\circledR}$, Stratagene). An IScel recognition site was engineered into the middle of the first intron in the rhodopsin gene at position 1340 from the 500 start of translation, but it was not used in the experiments described here. The targeting vector 501 was constructed in such a way that there is no Lox site between the MOPS promoter and the 502 rhodopsin transcription unit.

503 The Darwin Transgenic Core Facility, Baylor College of Medicine, electroporated ES cells and 504 injected correctly targeted ES cells (those selected for HPRT' ${ }^{+} \mathrm{TK}^{-}$genotype) into blastocysts 505 from albino C57BL/6-Tyr ${ }^{c-B r d}$ mice (43). Founder mice carrying the HPRT-P23H-hRho-TagRFP 506 allele were crossed to GDF-9-iCre mice (44) to remove the HPRT minigene and screened to 507 ensure germline transmission of the correct targeted sequence without HPRT. P23H-hRho508 TagRFP mice were extensively backcrossed to C57BL/6 mice. We validated that the knockin 509 was successful by sequencing genomic DNA from the knockin mouse. We verified expression 510 of the P23HhRhoRFP fusion by fluorescence microscopy of retinas and by immunoblotting (Fig. 511 1, Fig. 2).

512 The P23H-human-rhodopsin-RFP (P23H-RFP) knockin mice were generated by the BCM 513 Genetically Engineered Mouse core, using a strategy similar to the one previously described for 

selection (HAT medium) was carried out with a plasmid containing 5' and 3' sequences identical to flanking sequences of the mouse rhodopsin gene, an intervening sequence corresponding to

517 the human Rhodopsin gene encoding the $\mathrm{P} 23 \mathrm{H}$ mutation found in patients, a C-terminal fusion

518 with the fluorescent protein Tag RFP-T, a final C-terminal peptide corresponding to the C-

519 terminal 9 residues of human rhodopsin (TETSQVAPA, the epitope for the 1D4 monoclonal

520 antibody and a putative outer segment targeting signal), a STOP codon, an endogenous

521 polyadenylation signal, followed by an expression cassette (minigene) for human

522 hypoxanthinephosphoribosyltransferase (HPRT). The HPRT minigene was flanked by loxP

523 sites, so it could be looped out in vivo by passing through the germline of a Zp3Cre mouse expressing Cre recombinase in oocytes $(71,72)$.

The junction between the mouse and human $R H O$ sequences is at the Sacl site in the 5'UTR between the transcription start sites and the translation start sites. Unlike our P23H-Rho-

527 GFP knockin described previously (35), there was no lox site added to the 5'UTR, although

528 there is a lone loxP site remaining in the 3'-end following loop-out of the HPRT minigene. The

529 sequence of the P23H-hRho-TagRFPr allele was verified by Sanger sequencing. We generated

530 a new hRho-EGFP knockin mouse line with an additional C-terminal 1D4 epitope sequence tag

531 by a similar approach, using WT human rhodopsin sequence and EGFP coding sequence

532 instead of Tag-RFP-T. Generation of the original hRho-EGFP knockin line was described

533 previously (41)

Both P23H-RFP and hRho-EGFP-1D4 mice were extensively backcrossed to C57BL/6

535 (>10 generations). Wild-type (+/+) C57BL/6 littermates were used as controls throughout this

536 study. The following genotyping PCR primers were used for the P23HhRhoRFP knockin allele

537 (5'- GTTCCGGAACTGCATGCTCACCAC) and (5'- GGCGCTGCTCCTGGTGGG), which

538 generate a 975 kb knockin band and 194bp WT band. 
All animal research in this study was approved by the Institutional Animal Care and Use

541 set forth in the Statement for the Use of Animals in Ophthalmic and Vision Research of the

542 Association for Research in Vision and Ophthalmology (ARVO).

Western blotting

Retinal lysates were made by needle extruding mouse retinas in ice-cold Cracking buffer: $25 \mathrm{mM}$ Tris (pH 8), $300 \mathrm{mM}$ sucrose, $15 \mathrm{mM}$ EDTA, $2 \mathrm{mM} \mathrm{MgCl} 2+1 x$ protease inhibitor cocktail (GenDepot), and lysates were cleared with centrifugation. Protein concentration was calculated with the BCA assay (Bio-Rad), and sample application buffer was added to lysates, which were sonicated to reduce sample viscosity. $100 \mu \mathrm{g}$ of each lysate sample was loaded on $10 \%$ acrylamide gels for SDS-PAGE. Gels were transferred onto nitrocellulose in Tris-Glycine-

551 SDS buffer and probed with primary antibodies: anti-1D4 (Rho) at $1 \mu \mathrm{g} / \mathrm{ml}$, anti-RFP (Kerafast, 552 6a11f) at $1 \mu \mathrm{g} / \mathrm{ml}$, anti-beta-actin (Cell Signaling Technology, 8HD10) diluted 1:1000. Mouse monoclonal anti-1D4 (73) was purified in-house from hybridoma culture medium. Membranes were secondary labeled with one of the following secondary antibodies: anti-mouse or antirabbit IRDye680 (LI-COR Biosciences), diluted 1:10,000, and were imaged on a LI-COR were adjusted maintaining a linear slope.

\section{Retinal immunofluorescence}

For cyrosectioning, mouse eyes were enucleated and either 1) cornea punctured and

561 immersion fixed in 4\% paraformaldehyde (PFA) diluted in 1x PBS for 45 mins at room

562 temperature before the cornea and lens were removed in 1xPBS, or 2) the cornea and lens

563 were removed and 1xPBS and eye cups were directly embedded Optical Cutting Temperature

564 (OCT) media in plastic cryomolds and flash frozen on a floating liquid nitrogen platform. Fixed 
eye cups were cryoprotected in $30 \%$ sucrose before mounting in OCT in plastic molds and flash freezing. $8 \mu \mathrm{m}-10 \mu \mathrm{m}$ cryosections were collected on poly-L-lysine coated glass slides (EMS), and unfixed eye cups sections were immediately fixed with $2 \%$ PFA for 2 minutes. This light

568 fixation method was used for all immunolabeling experiments that included anti-centrin cilia

569 immunolabeling. Superior-inferior positions were marked in eyes to be used for retina thickness

570 measurements prior to enucleation to maintain proper orientation throughout fixation and

571 sectioning.

For immunohistochemistry, sections were blocked with either $2 \%$ normal goat serum

573 (NGS) (Fitzgerald), 2\% bovine serum albumin (BSA) (Sigma), 2\% fish skin gelatin (FSG)

574 (Sigma), 0.2\% saponin diluted in 1x PBS, or with SUPER block: 15\% NGS, 5\% BSA, 5\% BSA-c

575 (Aurion), 5\% FSG, 0.2\% saponin in 1xPBS (sections from Fig 7C-D, Fig 9, Fig 10). Blocked

576 sections were probed with $0.5 \mu \mathrm{g}-2 \mu \mathrm{g}$ of primary antibodies in the same blocking buffer overnight at room temperature, protected from light. The following antibodies were used: mouse anti-centrin (20H5) (EMD Millipore, 04-1624); rabbit anti-centrin 2 (Proteintech, 15877-1-AP); rabbit anti-BiP (Abcam, ab21685); mouse anti-KDEL (10C3) (Sigma-Aldrich, 420400); mouse anti-GM130 (35/GM130) (BD, 610822); rabbit anti-cone arrestin (EMD Millipore, AB15282). mouse/anti-rabbit IgG Alexa 555 (Thermo Fisher), or F(ab')2-goat anti-mouse/anti-rabbit IgG Alexa 647 (Thermo Fisher), diluted 1:500 in blocking buffer, for $1-1.5$ hours at room

585 temperature, protected from light. Sections were counterstained with $0.3 \mu \mathrm{M}$ DAPI for 1 hour 586 protected from light. Widefield and confocal sections were mounted with \#1.5 coverslips in 587 VECTASHIELD (Vector Laboratories), and SIM sections were mounted in ProLong Glass 588 (Thermo Fisher). 
591 Photometrics CoolSnap cf Photometrics digital camera (Roper Scientific) and excitation via a

592 mercury lamp using dichroic mirrors and filters for excitation and emission wavelength selection.

593 Full retina sections were generated by merging overlapping captures in Fiji/lmageJ using the

594 "Stitching" plugin $(74,75)$. ONL thickness was measured from these full retina section files in

$595 \mathrm{Fiji} /$ ImageJ. The position of the optic nerve was designated as position "0"; we then traced the

596 DAPI+ ONL along the superior (positive $\mathrm{nm}$ positions) and inferior (negative $\mathrm{nm}$ positions)

597 retina. At each position to be measured, we drew a rectangular Region of Interest with edges at

598 the top and bottom of the ONL with a constant perpendicular length of $50 \mu \mathrm{m}$ at each position

599 and collected ONL thickness measurements.

600

Confocal imaging was performed on either a Leica TCS-SP5 laser scanning confocal

601 microscope with a 63x oil immersion objective (Leica, HC PL APO CS2 63.0x, numerical

602

aperture 1.40) or a Zeiss LSM710 laser scanning microscopy with a 63x oil immersion objective

603 (Zeiss Plan Apo 63.0x, numerical aperture 1.40). On both systems, sequential imaging scans

604 with 405 diode, $488 \mathrm{~nm}$ argon, $543 \mathrm{~nm}$ HeNe, and $633 \mathrm{~nm}$ HeNe lasers were performed with

605

parameters set to capture sub-saturation fluorescence and to avoid cross-talk. Z-stacks of $1 \mu \mathrm{m}$

606

optical confocal sections were projected in Fiji/lmageJ based on maximum intensity values.

607

SIM imaging was performed on a DeltaVision OMX Blaze Imaging System (v4) (GE

608

Healthcare) with a PLANPON6 60x / NA 1.42 (Olympus) oil immersion objective using oil with a refractive index of 1.520 . The system features $488 \mathrm{~nm}, 568 \mathrm{~nm}$ and $647 \mathrm{~nm}$ laser lines, and a front illuminated Edge sCMOS (PCO) camera. Images were captured in sequential SIM mode

611 with 15 fringe shift images acquired per optical section per channel. SIM reconstruction was

612 subsequently performed in softWoRx 7 software. Z stacks of $125 \mathrm{~nm}$ optical SIM sections were 613 projected in Fiji/lmageJ based on max intensity values.

All images were pseudo-colored and processed for clarity in Fiji/lmageJ; minimum and

615 maximum input values were adjusted maintaining a linear slope. Magnified images of rod cilia

616 were digitally straightened with the Straighten tool in Fiji/lmageJ. 
Mice were dark adapted overnight and anesthetized with $90 \mathrm{mg} / \mathrm{kg}$ ketamine $+14 \mathrm{mg} / \mathrm{kg}$

620 xylazine. $0.5 \%$ tropicamide was added as a mydriatic to both eyes with $2.5 \%$ phenylephrine

621 hydrochloride and 0.5\% proparacaine hydrochloride for analgesia/anesthesia. $2.5 \%$

622 methylcellulose was used to maintain conductivity and for corneal hydration. A ground electrode

623 was inserted into the mouse forehead, and wire electrode loops were placed over each eye. We

624 used the UTAS BigShot Visual Electrodiagnostic System (LKC Technologies) for ERG

recordings. Mice were placed within a Ganzfeld chamber and responses were recorded per a

627 flashes for the intensity range of $-55 \mathrm{~dB}$ to $-10 \mathrm{~dB}$ at $5 \mathrm{~dB}$ increments. Photopic recordings were

629 Recordings were averaged from 60 flashes for $-10 \mathrm{~dB}$ and $0 \mathrm{~dB}$ intensities, and 20 flashes for 5

on the instrument's calibration data. post-flash. Implicit time was the time in ms between the a-wave to the b-wave values. Photopic

637 b-wave waves were also filtered and were baseline to the maximum between $30-120$ ms post-

638 flash.

\section{Transmission Electron Microscopy}

The following TEM preparation, based on (8), enhances staining and contrast of internal

642 cell membranes in rod photoreceptors. Mouse eyes for TEM were cornea punctured in the 
643

644

645

646

647

648

649

650

651

652

653

654

655

656

657

658

659

660

661

662

663

664

665

666

667

668

following ice-cold TEM fixative: 2\% PFA, 2\% glutaraldehyde (EMS), $2.2 \mathrm{mM} \mathrm{CaCl}_{2}$ diluted in 50 mM MOPS buffer ( $\mathrm{pH} 7.4)$ and were then immersion fixed in the same fixative for either 1) 45

mins at room temperature with gentle agitation followed by cornea and lens removal and another 1.5 hours on ice, or 2) $4^{\circ} \mathrm{C}$ overnight before cornea and lens removal. Fixed eye cups were embedded in $4 \%$ low melt agarose, and $150 \mu \mathrm{m}$ vibratome sections were cut along the longitudinal plane. Sections were fixed in $1 \%$ tannic acid (EMS), $0.5 \%$ saponin (Calbiochem) diluted in $0.1 \mathrm{M}$ HEPES ( $\mathrm{pH} 7.3$ ) for 1 hour at room temperature with gentle rocking. After rinsing again, sections were stained with $1 \%$ uranyl acetate (EMS) diluted in 0.1 maleate buffer $(\mathrm{pH}$ ) for 1 hour at room temperature with gentle rocking.

Sections were then dehydrated in the following ethanol series: $50 \%, 70 \%, 90 \%, 100 \%$, $100 \%$, in half dram glass vials filled with $1 \mathrm{~mL}$ of dehydrant. For Eponate 12 embedding, sections were additionally dehydrated in $100 \%$ acetone $2 x$, for 15 minutes. Using the solutions of the PELCO Eponate 12 kit with DMP-30 (Ted Pella), a medium hardness resin mix (without accelerator) was prepared and mixed. After dehydrating, the sections were embedded in stages of Eponate 12 resin mix to acetone for the following incubation times: $50 \%: 50 \%$ (5 hours), 75\%:25\% (overnight, 16-20 hours), 100\% resin mix (8 hours), 100\% resin mix (overnight, 16-20 hours). The DMP-30 accelerator was added to the Eponate 12 resin mix just before mixing or incubating. All embedding steps were incubated on a room temperature roller set to a slow speed.

Resin embedded sections were then mounted in full resin either between two sheets of ACLAR Film (EMS) or in an inverted BEEM embedding capsules (EMS). Mounted sections were cured at $65^{\circ} \mathrm{C}$ for at least 48 hours. $70-100 \mathrm{~nm}$ ultrathin resin sections were cut on a Leica UC6 ultramicrotome with a Diatome diamond knife. Ultrathin sections were collected onto cleaned 100 mesh copper grids (EMS). Grids for TEM were post-stained on glue sticks first in $1.2 \%$ uranyl acetate (diluted in Milli-Q water) for 4-6 minutes, then in Sato's Lead for 4-6 minutes after rinsing in boiled Milli-Q water and drying. Grids were rinsed again and dried before 
storage. Imaging was performed on either a Hitachi H7500 TEM or JEOL JEM-1400 at magnifications up to 25000x. TEM images were processed for clarity in Fiji/lmageJ by adjusting the minimum and maximum input values (maintaining a linear slope) for contrast and with the Straighten tool.

\section{$R N A$ extraction and relative quantification of $m R N A$}

Six pairs of retinas from three postnatal day 30 (P30) WT C57BL/6 mice and three P30 P23H-RFP/+ heterozygous mice were collected and homogenized for RNA extraction. Directzol $^{\text {TM }}$ RNA MiniPrep Kit (Genesee Scientific/Zymo Research, Catalog No: R2051) was used for RNA extraction and $25 \mu$ l of DNase/RNase free was used for elution. RNA extraction product was diluted 20-fold in DNase/RNase free water (from Zymo Research Direct-zol ${ }^{\mathrm{TM}}$ RNA MiniPrep Kit) before recording the UV absorbance spectra on a Hewlett Packard 8452 Diode Array Spectrophotometer. Only RNA samples with $1.7 \leq A_{260} / \mathrm{A}_{280}$ ratio $\leq 2.0$ was used for Reverse Transcription Polymerase Chain Reaction (RT-PCR) experiments. $5 \mu$ of the RNA extraction product was run on a $1 \%$ agarose gel to examine purity. RNA samples with $28 \mathrm{~S} / 18 \mathrm{~S}$ ratio $\geq 2$ were used for analysis. The total RNA input for each RT-PCR reaction was $500 \mathrm{ng}$. RT-PCR was performed using LunaScript RT SuperMix Kit (New England Biolabs - Catalog No: E3010L). Each Complementary DNA (cDNA) sample from RT-PCR was diluted 100-fold prior to quantitative Polymerase Chain Reaction (qPCR). In the Amplifyt ${ }^{\mathrm{TM}}$ 96-Well PCR Plate (Thomas Scientific, Catalog No: 1148B05), 4 ul of the diluted cDNA was added per qPCR reaction followed by 16 ul of master mix with target gene qPCR primers (See primer nucleotide sequences in Table 1), Luna® Universal qPCR Master Mix (New England Biolabs, Catalog No: M3003L) and DEPC treated water. Per qPCR run, three technical replicates were run per biological replicate. Plates were sealed by LightCycler® 480 Seal Foil (Roche Life Science, Catalog No: 04729757 001) then spun down in a mini plate spinner. The qPCR reactions were carried out using the $\mathrm{C} 1000$ Touch $^{\mathrm{TM}}$ Thermal Cycler (BioRad) and the SYBR fluorescent data 
695 collected using the CFX96 Optical Reaction Module for Real-Time PCR Systems (BioRad).

696 Reactions with primers specific for the genes encoding HPRT1 and RPL19 were carried out in

697 parallel for normalization. Data were extracted from CFX BioRad Manager 3.1 as 2007 Excel

698 files. $C_{q}$ values from 3 technical replicates (with standard deviation $\leq 0.25$ ) were averaged then

699 converted to Relative Quantification Value (RQ value) following Livak Method. Graph design

700 and statistical significance analysis of calculated RQ values were carried out using GraphPad

701 Prism 8.4.3. A 95\% confidence interval for a two-tailed t-Test was used as the criterion for

702 statistical significance. $P$ values for the two-tailed t-tests are reported. 
Table 1: Q-RTPCR Primer Nucleotide Sequences:

\begin{tabular}{|c|c|c|c|}
\hline Gene & Forward Primer & Reverse Primer & Source* \\
\hline HPRT & 5'-CAGTCCCAGCGTCGTGATTA-3' & 5'-TGGCCTCCCATCTCCTTCAT-3' & \\
\hline RPL19 & 5'-ATGCCAACTCCCGTCAGCAG-3' & 5'-TCATCCTTCTCATCCAGGTCACC-3' & (77) \\
\hline $\mathrm{BiP}$ & 5'-ACTTGGGACCACCTATTCCT-3' & 5'-ATCGCCAATCAGACGCTCC-3' & (78) \\
\hline DERL1 & 5'-CGCGATTTAAGGCCTGTTAC-3' & 5'-GGTAGCCAGCGGTACAAAAA-3' & (77) \\
\hline XBP1 & 5'-GAGTCCGCAGCAGGTG-3' & 5'-GTGTCAGAGTCCATGGGA-3' & (77) \\
\hline $\mathrm{CHOP}$ & 5'-CTGGAAGCCTGGTATGAGGAT-3' & 5'-CAGGGTCAAGAGTAGTGAAGGT-3' & $(78)$ \\
\hline ATF6 & 5'-TGCCTTGGGAGTCAGACCTAT-3' & 5'-GCTGAGTTGAAGAACACGAGTC-3' & $(78)$ \\
\hline PERK & 5'-AGTCCCTGCTCGAATCTTCTT-3' & 5'-TCCCAAGGCAGAACAGATATACC-3' & (78) \\
\hline EIF2a & 5'-TATCCCAACTTTGCCGGACC-3' & 5'-AGGCTCCTGTCTTGTCAACC-3' & \\
\hline mRho & 5'-CCATGGCAGTTCTCCATGCT-3' & 5'-CATCTCCCAGTGATTCTTGCC-3' & \\
\hline mhRho & 5'-CCATGGCAGTTCTCCATGCT-3' & 5'-TTCTCCCCGAAGCGGAAGTT-3' & (62) \\
\hline
\end{tabular}

${ }^{*} A l l$ primer sequences not sourced from the literature were designed with Primer-BLAST (NCBI) for

specificity and to span exon-exon junctions. 
708 Statistical analysis

711 width with age was fit to a single exponential decay as $\mathrm{W}(\mathrm{t})=(\mathrm{W}(0)-\mathrm{W}(\infty)) \exp -(\mathrm{t} / \tau)+\mathrm{W}(\infty)$

712 Where $\mathrm{W}(\mathrm{t})$ is the measured width at age, $\mathrm{W}(\infty)$ is the plateau value to which it declines, $\mathrm{W}(0)$

713 is an initial value, and $\tau$ is the time constant. The data were fit to the equation with $\mathrm{W}(0), \mathrm{W}(\infty)$

714 and $\tau$ as floating fit parameters in a Marquardt-Levenberg least-squares fitting algorithm using

715 Prism®. 


\section{Acknowledgements}

718 The authors would like to thank Dr. Ching-Kang Jason Chen and Dr. Melina Agosto for help with

719 ERG recordings and analysis. This work was supported in part by NIH research grants R01-

720 EY01173, R01EY026545 and R01EY031949, core grants P30EY002520 and P30CA125123,

721 MAR was supported by a grant from the Knights Templar Eye Foundation and NIH grant F32-EY027171.

\section{Author Contributions}

725 MAR, FC, VN, LK and FH performed experiments, analyzed data and contributed to writing.

726 MAR wrote the original draft. TGW and JHW provided funding, supervised aspects of the

727 projects, analyzed data and edited the manuscript. 
Figure 1. Construction and validation of the P23H-hRho-TagRFP-T knockin mouse. (A)

732 Targeting construct used in embryonic stem cells and resulting gene structure after germline

733 transmission. (B) A map of the knock-in human P23H-rhodopsin-RFP fusion gene. A portion of 734 the sequence of exon 1 from the $\mathrm{P} 23 \mathrm{H}-\mathrm{Rho}$ allele in the fusion gene (blue) is aligned to the 735 same human wild-type (WT) Rho allele sequence (black). The mutated codon 23 is in bold font.

736 The transition sequence from the 1D4 terminal signal sequence to the Tag-RFP-T with a short 737 linker sequence is shown in the middle sequence panel and the C-terminal sequence with the extra 1D4 epitope sequence appended to the end Tag-RFP-T prior to the stop codon. (C) Western blot confirmation of the P23HhRhoRFP fusion protein expression in $\mathrm{P} 23 \mathrm{H}-\mathrm{RFP} /+$ heterozygous retinas. Retinal lysates are from a wild-type $(+/+)$ mouse, age $\mathrm{P} 22$, and a $\mathrm{P} 23 \mathrm{H}-$ $\mathrm{RFP} /+$ mouse, age $45 ; 100 \mu \mathrm{g}$ of total protein from each lysate was loaded onto SDS-PAGE gels. Blot membranes were probed with either of the following antibodies: anti-1D4, anti-RFP or anti-beta (ß)-actin (a loading control). Blot scans for the protein ladder were used to mark molecular weight sizes (in kilodaltons, $\mathrm{kDa}$ ) on the left of each blot image. The $\sim 65 \mathrm{kDa}$ $\mathrm{P} 23 \mathrm{HhRhoRFP}$ fusion protein band is present in the P23H-RFP/+ lane in both anti-1D4 and anti-RFP blot scans (magenta arrows). The monomeric mouse Rho protein band is in both lanes in the anti-1D4 blot scan (black arrow). Higher MW species are formed by rhodopsin multimerization.

Figure 2. Localization of RFP fluorescence in the P23H-hRho-RFP knockin mouse retina. (A) Widefield fluorescence images of mouse retinal cryosections from age-matched P30 wild-

751 type $(+/+)$, heterozygous (P23H-RFP/+) and homozygous knockin mice (P23H-RFP/P23H-

752 RFP). Sections were counterstained with DAPI to label nuclei in the retina (blue). RFP

753 fluorescence from the mutant P23HhRhoRFP fusion protein is shown in magenta. Magnified 754 regions from each section are insets, and the outer nuclear layer $(\mathrm{ONL})$ of each retina, which is 755 the location of the DAPI+ photoreceptor nuclei, is demarcated. (B) Confocal z-projection image 
756

757

758

759

760

761

762

763

764

765

766

767

768

769

770

771

772

773

774

775

776

777

778

779

780

781

of a retinal cryosection from a P30 heterozygous P23H-RFP/+ mouse. The brightest RFP signal is in the outer photoreceptor layers, the inner segment and outer segment region (IS/OS), where P23HhRhoRFP protein is localized in aggregates. In the same image, with the gain of the RFP signal raised to saturation, additional $\mathrm{P} 23 \mathrm{HhRhoRFP}$ protein is observed in the rod photoreceptor synapses within the outer plexiform layer (yellow arrows). To a lesser degree, $\mathrm{P} 23 \mathrm{HhRhoRFP}$ is also localized in the ONL, in the cytoplasm surrounding the photoreceptor nuclei (white arrows). (C) Confocal z-projection through a retinal cryosection from a P30 P23HRFP/P23H-RFP homozygote. The width of the DAPI-positive ONL is thinner compared to the heterozygote. In the homozygous retina, mutant P23HhRhoRFP fusion protein is also more prominently localized in the ONL compared to the heterozygote. INL = inner nuclear layer.

\section{Figure 3. P23HhRhoRFP+ fusion aggregates are localized in the inner segments of rod}

photoreceptor neurons. (A) In a confocal z-projection image of a retinal section from the hRho-GFP-1D4/P23H-RFP heterozygous mouse retina, at 3 weeks of age, the wild-type hRhoGFP fusion (green), which has an additional C-terminal 1D4 signal sequence, is correctly localized to the outer segments (OS) of rod neurons. In this retina, RFP+ aggregates containing mutant P23HhRhoRFP protein (magenta) are primarily located in the inner segments (IS) of rods and almost entirely segregated from the GFP+ OS layer. White dotted lines in the figure demarcate the OS/IS boundary and the IS/outer nuclear layer (ONL) boundary. (B) In SIM micrographs of retinal sections from the same heterozygous mouse line at age 6 weeks, GFP+ OS and RFP+ IS aggregates remain segregated with no apparent co-localization. (C) In SIM images of retinal sections from an alternate GFP/RFP heterozygote at age P30, in which the wild-type hRho-GFP fusion does not have an additional 1D4 signal peptide, the GFP

$$
\text { fluorescence is not exclusively located in the OS layer, but rather is partially mislocalized with }
$$

$$
\text { P23H-hRho-RFP. In a magnified example, hRho-GFP is co-localized around and within a }
$$

$$
\text { P23HhRhoRFP aggregate (yellow arrows). In another magnified example, hRho-GFP is }
$$

$$
\text { wrapped around an P23HhRhoRFP aggregate (white arrow). }
$$


Figure 4. Mutant P23H-hRho-RFP inner segment aggregates are localized near the basal

body in rod neurons. (A) In a SIM z-projection image of a retinal section from the P23H-RFP/+ mouse at age P14, centrin immunolabeling (green) marks the location of rod connecting cilium (CC) and basal body (BB) relative to the P23HhRhoRFP fluorescent aggregates (magenta). In magnified views, single RFP+ inner segment aggregates are located just proximal to the CC (yellow arrow) and BB (white stars). The BB region is demarcated by the centrin+ mother centriole at the proximal end of the $\mathrm{CC}$ and the daughter centriole, which is a separated centrin+ puncta beneath the mother centriole. (B) A SIM image of a retinal section from the P23H-RFP/+ mouse at age P30 also with centrin immunolabeling. In magnified views, the RFP+ aggregates at P30 are still generally located proximal to the BB (white stars), with some examples of RFP overlapping with the BB region. The organization of the RFP+ aggregates in these P30 rods are less compact and more reticulated compared to the examples from age P14 (see examples in A).

Figure 5.Time course of retinal degeneration in P23H-RFP/+ heterozygous and P23HRFP/+ homozygous mice. (A) Confocal z-projection images of retinal cryosections from wildtype (+/+), and P23H-RFP heterozygote (het) and P23H-RFP/P23H-RFP homozygous (homo) mice at various time points. DAPI (blue) labels photoreceptor nuclei in the outer nuclear layer $(\mathrm{ONL})$ and the bipolar cell nuclei in the inner nuclear layer (INL). RFP fluorescence (magenta) is primarily located in the inner segment layers of het retinas at all time points, while RFP is also located in the ONL in homo retinas. At P90, the homo ONL is reduced to very few disorganized nuclei surrounded by RFP. (B) Time course plot of ONL thickness between genotypes. Measurements correspond to the ONL thickness of the retina $500 \mu \mathrm{m}$ inferior to the optic nerve. Unfilled shapes correspond to the mean value, and error bars signify standard error of the mean. Solid lines represent fits to exponential decays to plateau, as described in Methods. Unpaired t-tests were calculated to compare $+/+$ and het values for significance. Comparisons with significant differences are: P61 +/+ vs P60 het ( $\left.{ }^{* *} p=0.0081\right), P 90+/+$ vs P90 het 

positions from the optic nerve to the inferior and superior retina. Positions directly adjacent to

811 the optic nerve position ("0") correspond to $100 \mu \mathrm{m}$ superior and inferior to the optic nerve. The

812 most peripheral positions of each plot correspond to $100 \mu \mathrm{m}$ from the superior and inferior ends

813 of the retina. For each position on the plots, circles correspond to mean values and error bars

814 signify standard error of the mean. (D) Corresponding spider plots for P14, P30, and P90 P23H-

815 RFP/+ het retinal cryosections. Tests for statistical differences between plots from different time

816 points were performed using Two-way ANOVA with Šídák multiple comparisons test. The only

817 test with significance is $\mathrm{P} 14$ het vs $\mathrm{P} 90$ het $\left({ }^{*} \mathrm{P}=0.0215\right)$. (E) Spider plots for $\mathrm{P} 23 \mathrm{H}-\mathrm{RFP}$

818 homozygous retinal cryosections at the same time points. Two-way ANOVA with Šídák multiple 819 comparison tests produced significant differences between all time points: P14 vs P30, P14 vs

82090 , and P30 vs P90 (all ${ }^{* * *} \mathrm{P}<0.0001$ ). (F) Spider plots for all retinal cryosections at timepoint

821 P30 to compare differences between genotypes. Two-way ANOVA with Šídák multiple

822 comparison tests produced significant differences at P30 between: +/+ vs homo and het vs

823 homo. (both ${ }^{* * * *} \mathrm{P}<0.0001$ ). (G) Spider plots for timepoint P90 to compare genotypes. Two-way

824 ANOVA with Šídák multiple comparison tests produced significant differences at P90 between:

$825+/+$ vs homo. and het. vs homo. (both $\left.{ }^{* * *} \mathrm{P}<0.0001\right)$.

826 Figure 6. P23H-RFP/+ heterozygous mice have slightly reduced rod photoreceptor

827 electroretinogram (ERG) responses. (A) Example ERG recordings from +/+ wild-type, $\mathrm{P} 23 \mathrm{H}-$

828 RFP/+ heterozygous (het) and P23H-RFP/P23H-RFP homozygous (homo) mice at age P30. (B)

829 Aggregate of P30 scotopic a-wave amplitudes. In all plots, solid shapes are data points, empty

830 shapes signify mean values, and error bars signify standard error of the mean. Statistical

831 comparison tests of all the ERG data were performed using Two-way ANOVA with Šídák

832 multiple comparisons tests. All statistical comparisons of P30 a-wave amplitudes were

833 significant: $+/+$ vs het $\left({ }^{* * *} \mathrm{P}<0.0001\right),+/+$ vs homo $\left({ }^{* * * *} \mathrm{P}<0.0001\right)$, and het vs homo 

not statistically different (P=0.27). (D) Aggregate of P30 scotopic b-wave amplitudes.

836 Statistically significant differences were calculated for $+/+$ vs homo $\left({ }^{* * *} P<0.0001\right)$ and het vs

837 homo $\left({ }^{* * *} \mathrm{P}<0.0001\right)$. WT vs het was not statistically different $(P=0.9744)(E)$ Scotopic $b$-wave 838 amplitudes between $\mathrm{P} 30$ and P90 P23H/+ het mice were not statistically different $(\mathrm{P}=0.9375)$.

839 (F) Aggregate implicit times, the time to peak scotopic b-wave post a-wave, in P30 +/+ and het 840 mice. P30 het mice had higher implicit times than $+/+$ at intermediate flash stages, but the

842 of P30 photopic b-wave amplitudes. There were no statistically significant differences among 843 the genotypes: $+/+$ vs het $(P=0.749),+/+$ vs homo $(P=0.1298)$, het vs homo $(P=0.1553)$.

Figure 7. Cone immunolocalization and TUNEL analysis of photoreceptor cell death in +/+, P23H-RFP/+ heterozygous (het), and P23H-RFP/P23H-RFP homozygous (homo) mice with TUNEL+ nuclei (green) within the DAPI stained nuclei of the ONL (blue). RFP fluorescence is magenta. (D) Time course plot of aggregate TUNEL+ nuclei/ $/ \mathrm{mm}^{2}$ measurements among all genotypes at multiple time points. Statistical comparisons among groups were performed using unpaired t-tests. At P14, homo retinas have statistically more TUNEL+ nuclei compared to both $+/+\left({ }^{*} \mathrm{P}=0.0167\right)$ and het $\left({ }^{* *} \mathrm{P}=0.0098\right)$ retinas. At $\mathrm{P} 30$, het retinas have statistically more

856 TUNEL + nuclear compared to $+/+$ retinas $\left({ }^{*} \mathrm{P}=0.042\right)$, but the rate of TUNEL+ nuclei between

857 het and $+/+$ retinas is not statistically different at age $P 90(P=0.312)$.

858 Figure 8. mRNA levels of ER stress and Unfolded Protein Response markers are near 
measurements of the indicated messages in RNA extracted from retinas of heterozygotes as compared to wild-type (+/+) (n=3 for both), normalized to HPRT (dark grey bars) or RPL19

862 (open bars). Statistical comparisons were made with two-tailed t-tests.: mhRho vs. RPL19 ${ }^{* *} \mathrm{P}=$

863 0.0099; mhRho vs. HPRT ${ }^{* * *} \mathrm{P}=0.000741$; $\mathrm{mRho}$ vs. RPL19 ${ }^{* * *} \mathrm{P}=0.000585$. mRho vs. HPRT

$864 \quad{ }^{* * *} \mathrm{P}=0.000542$

Figure 9. P23H-Rho-RFP/+ mutant rod photoreceptor neurons have distended inner

segments filled with ectopic membranes. (A-B) Conventional transmission electron

871 ( $n=19), P<0.0001$, unpaired t-test). Ectopic stacks of IS membranes in the swollen P23H-

$872 \mathrm{RFP} /+$ IS are marked with magenta arrows. Double-membraned autophagy compartments are

873 marked with magenta asterisks in the mutant P23H-RFP/+ IS. In magnified views of example

874 connecting cilia (CC) from each genotype, the length of the $\mathrm{CC}$ - measured by the densely

875 stained CC membrane - is indicated. In aggregate, the length of the CC in P23H-RFP/+ rods is

876 significantly greater than +/+ CC (het: $1.548 \mu \mathrm{m} \pm 0.206 \mu \mathrm{m}(\mathrm{sd})(\mathrm{n}=13) \mathrm{vs}+/+: 1.27 \mu \mathrm{m} \pm 0.247$

$877 \mu \mathrm{m}(\mathrm{sd})(\mathrm{n}=11), \mathrm{P}=0.0066$, unpaired t-test). (C) At age P30, the ectopic IS membranes in

$878 \mathrm{P} 23 \mathrm{H} /+$ rods appear more dysmorphic compared to P14 (magenta arrows). In addition, some

879 outer segments disc membranes within or adjacent to $\mathrm{P} 23 \mathrm{H}-\mathrm{RFP} /+$ rods with IS defects are also

880 disrupted and appear dysmorphic (green arrows). (D) In examples of the CC and basal OS

881 regions of $\mathrm{P} 30 \mathrm{P} 23 \mathrm{H} /+$ rods, the structure of the $\mathrm{CC}$ and basal body remain intact despite being 882 adjacent to ectopic IS membranes (magenta arrows); however, there is evidence that basal OS

883 disc morphogenesis is disrupted possibly due to OS axoneme instability (green arrows).

884 Figure 10. Mutant P23H-hRho-RFP protein is accumulated within ER membranes in P23H-

885 RFP/+ mouse rods. (A-B) Confocal z-projection images of a retinal cryosection from P14 
$\mathrm{P} 23 \mathrm{H}-\mathrm{RFP} /+$ and +/+ littermate mice, immunolabeled for either the ER lumenal marker BiP/GRP78 (green) (A) or the Golgi marker GM130 (green) (B). P23HhRhoRFP fluorescence is magenta. Co-localized BiP with RFP+ aggregates in the $\mathrm{P} 14 \mathrm{P} 23 \mathrm{H}-\mathrm{RFP} /+$ retina section is marked with white arrows. GM130+ Golgi membranes do not co-localize with RFP+ aggregates and appear unaffected in the P14 P23H-RFP/+ retina compared to +/+ sections. (C) SIM z-

891 projection images of a retina cryosection from an age $\mathrm{P} 30 \mathrm{P} 23 \mathrm{H}-\mathrm{RFP} /+$ mouse that is

892 immunolabeled for BiP (green) and centrin (white), which labels the connecting cilium (CC) and 893 basal body (BB). RFP fluorescence is magenta. In a magnified inner segment (IS), BiP colocalization with a P23HhRhoRFP aggregate is marked with white arrows. BiP+ ER near the cilium that is not colocalized with RFP is marked with yellow arrows. (D) Within the same SIM images, the $\mathrm{BiP}+\mathrm{ER}$ tightly surrounds a P23HhRhoRFP aggregate (white arrows). (E) In age P30 +/+ SIM control images, BiP immunolabeling marks the inner segment ER network that leads to the cilium. (F) SIM z-projections of age P30 P23H-RFP/+ retinal sections immunolabeled for KDEL, another ER lumen marker (green), along with centrin (white) and RFP (magenta). KDEL puncta are more diffuse than $\mathrm{BiP}$, but the localization pattern in the IS is similar; KDEL co-localization with the P23H-RFP aggregates (white arrows) and KDEL+ ER near the basal body that is not associated with RFP (yellow arrows) are labeled. (G) In age P30 +/+ SIM control images, KDEL immunolabeling (green) labels puncta throughout the inner segment. (H) SIM images of a P23H-RFP/+ retina cryosection at age P30 immunolabeled for GM130, a Golgi membrane marker (green), along with centrin (white) and RFP (magenta). Overall, The Golgi is proximally localized to the RFP+ aggregates at P30, but in some examples 907 the Golgi network reaches the centrin+ cilium. On a subcellular scale, the Golgi membranes are 908 generally segregated from RFP aggregates (yellow arrows), but small pieces of Golgi 909 membrane are found co-localized with some RFP aggregates (white arrows). (I) In age P30 +/+ 910 SIM control images of retinal cryosections, the Golgi is predominantly dissociated from the cilia; 911 however, examples of +/+ rods with GM130+ Golgi membrane networks that reach the basal 
912 body are shown. Gray dotted arrows throughout mark a region that is magnified from the same

913 image.

914

Figure 11. Localization of P23HhRh-RFP protein with the ER in the outer plexiform layer

916 (OPL) of P23H-RFP/+ retinas. (A) A SIM z-projection image of a P23H-RFP/+ retinal

917 cryosection, age P30, immunolabeled for BiP/GRP78 to label the ER (green) in the OPL and

918 surrounding area. P23HRhoRFP protein (magenta) fills the rod photoreceptor synapses in this

919 region. $\mathrm{BiP}+\mathrm{ER}$ labeling is present throughout the OPL and the outer nuclear layer (ONL). In a

920 magnified view of a pair of synapses, $\mathrm{P} 23 \mathrm{HhRhoRFP}$ protein is co-localized with the BiP+ ER

921 staining. (B) In a similar SIM magnified view of a P30 P23H-RFP/+ retina section

922 immunostained for KDEL (green), P23HRhoRFP protein is also co-localized with KDEL+ ER

923 staining. (C) As a control SIM image, a P30 P23H-RFP/+ retina section is immunostained for

924 GM130 to label Golgi. No Golgi membranes are evident in the OPL; however, they are present

925 in the inner nuclear layer (INL). 


\section{References}

938 1. Daiger SP, Sullivan LS, Bowne SJ. Genes and mutations causing retinitis pigmentosa. Clin Genet. 2013;84(2):132-41.

940 2. Hamel C. Retinitis pigmentosa. Orphanet journal of rare diseases. 2006;1:40.

941 3. Frederick JM, Hanke-Gogokhia C, Ying G, Baehr W. Diffuse or hitch a ride: how

942 photoreceptor lipidated proteins get from here to there. Biol Chem. 2020;401(5):573-84.

943 4. Palczewski K. G protein-coupled receptor rhodopsin. Annu Rev Biochem. 2006;75:743-

94467.

$945 \quad 5 . \quad$ Bok D. Retinal photoreceptor-pigment epithelium interactions. Friedenwald lecture.

946 Invest Ophthalmol Vis Sci. 1985;26(12):1659-94.

9476 6evany BM, Palczewski K. Phagocytosis of retinal rod and cone photoreceptors.

948 Physiology (Bethesda). 2010;25(1):8-15.

949 7. Steinberg RH, Fisher SK, Anderson DH. Disc morphogenesis in vertebrate

950 photoreceptors. J Comp Neurol. 1980;190(3):501-8.

951 8. Ding JD, Salinas RY, Arshavsky VY. Discs of mammalian rod photoreceptors form

952 through the membrane evagination mechanism. J Cell Biol. 2015;211(3):495-502.

9539 9. Roepman R, Wolfrum U. Protein networks and complexes in photoreceptor cilia. Subcell

954 Biochem. 2007;43:209-35.

955 10. Wensel TG, Potter VL, Moye A, Zhang Z, Robichaux MA. Structure and dynamics of 956 photoreceptor sensory cilia. Pflugers Arch. 2021.

957 11. Nachury MV. The molecular machines that traffic signaling receptors into and out of cilia.

958 Curr Opin Cell Biol. 2018;51:124-31.

959 12. Pearring JN, Salinas RY, Baker SA, Arshavsky VY. Protein sorting, targeting and

960

961

962

963

964

965

966

967

968

969

970

971

972

973

974

975

976

977

978

979

980 trafficking in photoreceptor cells. Prog Retin Eye Res. 2013;36:24-51.

13. Athanasiou D, Aguila M, Bellingham J, Li W, McCulley C, Reeves PJ, et al. The molecular and cellular basis of rhodopsin retinitis pigmentosa reveals potential strategies for therapy. Progress in Retinal and Eye Research. 2018;62:1-23.

14. Dryja TP, McGee TL, Reichel E, Hahn LB, Cowley GS, Yandell DW, et al. A point mutation of the rhodopsin gene in one form of retinitis pigmentosa. Nature. 1990;343(6256):3646.

15. Sullivan LS, Bowne SJ, Birch DG, Hughbanks-Wheaton D, Heckenlively JR, Lewis RA, et al. Prevalence of disease-causing mutations in families with autosomal dominant retinitis pigmentosa: a screen of known genes in 200 families. Invest Ophthalmol Vis Sci. 2006;47(7):3052-64.

16. Kaushal S, Khorana HG. Structure and Function in Rhodopsin. 7. Point Mutations Associated with Autosomal Dominant Retinitis Pigmentosa. Biochemistry. 1994;33(20):6121-8.

17. Saliba RS, Munro PM, Luthert PJ, Cheetham ME. The cellular fate of mutant rhodopsin: quality control, degradation and aggresome formation. J Cell Sci. 2002;115(Pt 14):2907-18.

18. Sung $\mathrm{CH}$, Schneider BG, Agarwal N, Papermaster DS, Nathans J. Functional heterogeneity of mutant rhodopsins responsible for autosomal dominant retinitis pigmentosa. Proc Natl Acad Sci U S A. 1991;88(19):8840-4.

19. Lewin AS, Drenser KA, Hauswirth WW, Nishikawa S, Yasumura D, Flannery JG, et al. Ribozyme rescue of photoreceptor cells in a transgenic rat model of autosomal dominant retinitis pigmentosa. Nat Med. 1998;4(8):967-71.

981 20. Olsson JE, Gordon JW, Pawlyk BS, Roof D, Hayes A, Molday RS, et al. Transgenic 982 mice with a rhodopsin mutation (Pro23His): a mouse model of autosomal dominant retinitis 983 pigmentosa. Neuron. 1992;9(5):815-30.

984 21. Ross JW, Fernandez de Castro JP, Zhao J, Samuel M, Walters E, Rios C, et al. 985 Generation of an inbred miniature pig model of retinitis pigmentosa. Invest Ophthalmol Vis Sci. 986 2012;53(1):501-7. 
1016

1017

1018

1019

1020

1021

1022

1023

1024

1025

1026

1027

1028

1029

1030

1031

1032

1033

1034

1035

22. Tam BM, Moritz OL. Characterization of rhodopsin $\mathrm{P} 23 \mathrm{H}$-induced retinal degeneration in a Xenopus laevis model of retinitis pigmentosa. Invest Ophthalmol Vis Sci. 2006;47(8):3234-41. 23. Bogea TH, Wen RH, Moritz OL. Light Induces Ultrastructural Changes in Rod Outer and Inner Segments, Including Autophagy, in a Transgenic Xenopus laevis P23H Rhodopsin Model of Retinitis Pigmentosa. Invest Ophthalmol Vis Sci. 2015;56(13):7947-55.

24. Santhanam A, Shihabeddin E, Atkinson JA, Nguyen D, Lin YP, O'Brien J. A Zebrafish Model of Retinitis Pigmentosa Shows Continuous Degeneration and Regeneration of Rod Photoreceptors. Cells. 2020;9(10).

25. Olsson JE, Gordon JW, Pawlyk BS, Roof D, Hayes A, Molday RS, et al. Transgenic mice with a rhodopsin mutation (Pro23His): A mouse model of autosomal dominant retinitis pigmentosa. Neuron. 1992;9(5):815-30.

26. Liu X, Wu TH, Stowe S, Matsushita A, Arikawa K, Naash MI, et al. Defective phototransductive disk membrane morphogenesis in transgenic mice expressing opsin with a mutated N-terminal domain. J Cell Sci. 1997;110 ( Pt 20):2589-97.

27. Wu TH, Ting TD, Okajima TIL, Pepperberg DR, Ho YK, Ripps H, et al. Opsin localization and rhodopsin photochemistry in a transgenic mouse model of retinitis pigmentosa.

Neuroscience. 1998;87(3):709-17.

28. Sakami S, Maeda T, Bereta G, Okano K, Golczak M, Sumaroka A, et al. Probing mechanisms of photoreceptor degeneration in a new mouse model of the common form of autosomal dominant retinitis pigmentosa due to $\mathrm{P} 23 \mathrm{H}$ opsin mutations. J Biol Chem. 2011;286(12):10551-67.

29. Naash MI, Hollyfield JG, al-Ubaidi MR, Baehr W. Simulation of human autosomal dominant retinitis pigmentosa in transgenic mice expressing a mutated murine opsin gene. Proc Natl Acad Sci U S A. 1993;90(12):5499-503.

30. Frederick JM, Krasnoperova NV, Hoffmann K, Church-Kopish J, Ruther K, Howes K, et al. Mutant rhodopsin transgene expression on a null background. Invest Ophthalmol Vis Sci. 2001;42(3):826-33.

31. Roof DJ, Adamian M, Hayes A. Rhodopsin accumulation at abnormal sites in retinas of mice with a human P23H rhodopsin transgene. Invest Ophthalmol Vis Sci. 1994;35(12):4049-

62.

32. Kakavand K, Jobling AI, Greferath U, Vessey KA, de longh RU, Fletcher EL.

Photoreceptor Degeneration in Pro23His Transgenic Rats (Line 3) Involves Autophagic and Necroptotic Mechanisms. Front Neurosci. 2020;14:581579.

33. LaVail MM, Nishikawa S, Steinberg RH, Naash MI, Duncan JL, Trautmann N, et al. Phenotypic characterization of $\mathrm{P} 23 \mathrm{H}$ and $\mathrm{S} 334$ ter rhodopsin transgenic rat models of inherited retinal degeneration. Exp Eye Res. 2018;167:56-90.

34. Sakami S, Kolesnikov AV, Kefalov VJ, Palczewski K. P23H opsin knock-in mice reveal a novel step in retinal rod disc morphogenesis. Hum Mol Genet. 2014;23(7):1723-41.

35. Price BA, Sandoval IM, Chan F, Simons DL, Wu SM, Wensel TG, et al. Mislocalization and Degradation of Human P23H-Rhodopsin-GFP in a Knockin Mouse Model of Retinitis Pigmentosa. Invest Ophthalmol Vis Sci. 2011;52(13):9728-36.

36. Shaner NC, Lin MZ, McKeown MR, Steinbach PA, Hazelwood KL, Davidson MW, et al. Improving the photostability of bright monomeric orange and red fluorescent proteins. Nat Methods. 2008;5(6):545-51.

37. Grassart A, Cheng AT, Hong SH, Zhang F, Zenzer N, Feng Y, et al. Actin and dynamin2 dynamics and interplay during clathrin-mediated endocytosis. J Cell Biol. 2014;205(5):721-35.

38. Boucrot E, Pick A, Camdere G, Liska N, Evergren E, McMahon HT, et al. Membrane fission is promoted by insertion of amphipathic helices and is restricted by crescent BAR domains. Cell. 2012;149(1):124-36. 
1036

1037

1038

1039

1040

1041

1042

1043

1044

1045

1046

1047

1048

1049

1050

1051

1052

1053

1054

1055

1056

1057

1058

1059

1060

1061

1062

1063

1064

1065

1066

1067

1068

1069

1070

1071

1072

1073

1074

1075

1076

1077

1078

1079

1080

1081

1082

1083

1084

1085

39. Weber J, Kabakci Z, Chaurasia S, Brunner E, Lehner CF. Chromosome separation during Drosophila male meiosis I requires separase-mediated cleavage of the homolog conjunction protein UNO. PLoS Genet. 2020;16(10):e1008928.

40. Lodowski KH, Lee R, Ropelewski P, Nemet I, Tian G, Imanishi Y. Signals governing the trafficking and mistrafficking of a ciliary GPCR, rhodopsin. J Neurosci. 2013;33(34):13621-38.

41. Chan F, Bradley A, Wensel TG, Wilson JH. Knock-in human rhodopsin-GFP fusions as mouse models for human disease and targets for gene therapy. Proc Natl Acad Sci U S A. 2004;101(24):9109-14.

42. Chan F, Hauswirth WW, Wensel TG, Wilson JH. Efficient mutagenesis of the rhodopsin gene in rod photoreceptor neurons in mice. Nucleic Acids Res. 2011;39(14):5955-66.

43. Zheng B, Sage M, Sheppeard EA, Jurecic V, Bradley A. Engineering mouse chromosomes with Cre-loxP: range, efficiency, and somatic applications. Mol Cell Biol. 2000;20(2):648-55.

44. Lan ZJ, Xu X, Cooney AJ. Differential oocyte-specific expression of Cre recombinase activity in GDF-9-iCre, Zp3cre, and Msx2Cre transgenic mice. Biol Reprod. 2004;71(5):1469-74. 45. Trojan P, Krauss N, Choe HW, Giessl A, Pulvermuller A, Wolfrum U. Centrins in retinal photoreceptor cells: regulators in the connecting cilium. Prog Retin Eye Res. 2008;27(3):237-59. 46. Robichaux MA, Potter VL, Zhang Z, He F, Liu J, Schmid MF, et al. Defining the layers of a sensory cilium with STORM and cryoelectron nanoscopy. Proc Natl Acad Sci U S A. 2019;116(47):23562-72.

47. Gorbatyuk MS, Starr CR, Gorbatyuk OS. Endoplasmic reticulum stress: New insights into the pathogenesis and treatment of retinal degenerative diseases. Prog Retin Eye Res. 2020;79:100860.

48. Chan P, Stolz J, Kohl S, Chiang WC, Lin JH. Endoplasmic reticulum stress in human photoreceptor diseases. Brain Res. 2016;1648(Pt B):538-41.

49. Athanasiou D, Aguilà M, Bevilacqua D, Novoselov SS, Parfitt DA, Cheetham ME. The cell stress machinery and retinal degeneration. FEBS Lett. 2013;587(13):2008-17.

50. Lin JH, Lavail MM. Misfolded proteins and retinal dystrophies. Adv Exp Med Biol. 2010;664:115-21.

51. Newton F, Megaw R. Mechanisms of Photoreceptor Death in Retinitis Pigmentosa. Genes (Basel). 2020;11(10).

52. Wensel TG, Gross AK, Chan F, Sykoudis K, Wilson JH. Rhodopsin-EGFP knock-ins for imaging quantal gene alterations. Vision Res. 2005;45(28):3445-53.

53. Lin JH, Li H, Yasumura D, Cohen HR, Zhang C, Panning B, et al. IRE1 signaling affects cell fate during the unfolded protein response. Science. 2007;318(5852):944-9.

54. Gorbatyuk MS, Knox T, LaVail MM, Gorbatyuk OS, Noorwez SM, Hauswirth WW, et al. Restoration of visual function in $\mathrm{P} 23 \mathrm{H}$ rhodopsin transgenic rats by gene delivery of BiP/Grp78. Proc Natl Acad Sci U S A. 2010;107(13):5961-6.

55. Aguila M, Bellingham J, Athanasiou D, Bevilacqua D, Duran Y, Maswood R, et al. AAVmediated ERdj5 overexpression protects against $\mathrm{P} 23 \mathrm{H}$ rhodopsin toxicity. Hum Mol Genet. 2020;29(8):1310-8.

56. Chiang WC, Kroeger H, Sakami S, Messah C, Yasumura D, Matthes MT, et al. Robust Endoplasmic Reticulum-Associated Degradation of Rhodopsin Precedes Retinal Degeneration. Mol Neurobiol. 2015;52(1):679-95.

57. Lobanova ES, Finkelstein S, Li J, Travis AM, Hao Y, Klingeborn M, et al. Increased proteasomal activity supports photoreceptor survival in inherited retinal degeneration. Nat Commun. 2018;9(1):1738.

58. Wen RH, Stanar P, Tam B, Moritz OL. Autophagy in Xenopus laevis rod photoreceptors is independently regulated by phototransduction and misfolded $\mathrm{RHO}(\mathrm{P} 23 \mathrm{H})$. Autophagy. 2019;15(11):1970-89. 
1086

1087

1088

1089

1090

1091

1092

1093

1094

1095

1096

1097

1098

1099

1100

1101

1102

1103

1104

1105

1106

1107

1108

1109

1110

1111

1112

1113

1114

1115

1116

1117

1118

1119

1120

1121

1122

1123

1124

1125

1126

1127

1128

1129

1130

1131

1132

1133

1134

1135

59. Karam A, Tebbe L, Weber C, Messaddeq N, Morle L, Kessler P, et al. A novel function of Huntingtin in the cilium and retinal ciliopathy in Huntington's disease mice. Neurobiol Dis. 2015;80:15-28.

60. Omori Y, Chaya T, Katoh K, Kajimura N, Sato S, Muraoka K, et al. Negative regulation of ciliary length by ciliary male germ cell-associated kinase (Mak) is required for retinal photoreceptor survival. Proc Natl Acad Sci U S A. 2010.

61. Comitato A, Schiroli D, Montanari M, Marigo V. Calpain Activation Is the Major Cause of Cell Death in Photoreceptors Expressing a Rhodopsin Misfolding Mutation. Mol Neurobiol. 2020;57(2):589-99.

62. Mao H, James T, Jr., Schwein A, Shabashvili AE, Hauswirth WW, Gorbatyuk MS, et al. AAV delivery of wild-type rhodopsin preserves retinal function in a mouse model of autosomal dominant retinitis pigmentosa. Hum Gene Ther. 2011;22(5):567-75.

63. Cideciyan AV, Sudharsan R, Dufour VL, Massengill MT, Iwabe S, Swider M, et al. Mutation-independent rhodopsin gene therapy by knockdown and replacement with a single AAV vector. Proc Natl Acad Sci U S A. 2018;115(36):E8547-E56.

64. $\quad$ Li P, Kleinstiver BP, Leon MY, Prew MS, Navarro-Gomez D, Greenwald SH, et al. AlleleSpecific CRISPR-Cas9 Genome Editing of the Single-Base P23H Mutation for RhodopsinAssociated Dominant Retinitis Pigmentosa. CRISPR J. 2018;1:55-64.

65. Latella MC, Di Salvo MT, Cocchiarella F, Benati D, Grisendi G, Comitato A, et al. In vivo Editing of the Human Mutant Rhodopsin Gene by Electroporation of Plasmid-based CRISPR/Cas9 in the Mouse Retina. Molecular therapy Nucleic acids. 2016;5(11):e389.

66. Giannelli SG, Luoni M, Castoldi V, Massimino L, Cabassi T, Angeloni D, et al. Cas9/sgRNA selective targeting of the $\mathrm{P} 23 \mathrm{H}$ Rhodopsin mutant allele for treating retinitis pigmentosa by intravitreal AAV9.PHP.B-based delivery. Hum Mol Genet. 2018;27(5):761-79. 67. Yao J, Qiu Y, Frontera E, Jia L, Khan NW, Klionsky DJ, et al. Inhibiting autophagy reduces retinal degeneration caused by protein misfolding. Autophagy. 2018;14(7):1226-38. 68. Comitato A, Di Salvo MT, Turchiano G, Montanari M, Sakami S, Palczewski K, et al. Dominant and recessive mutations in rhodopsin activate different cell death pathways. Hum Mol Genet. 2016;25(13):2801-12.

69. Nakamura PA, Shimchuk AA, Tang S, Wang Z, DeGolier K, Ding S, et al. Small molecule Photoregulin3 prevents retinal degeneration in the Rho(P23H) mouse model of retinitis pigmentosa. Elife. 2017;6.

70. Vent-Schmidt RYJ, Wen RH, Zong Z, Chiu CN, Tam BM, May CG, et al. Opposing Effects of Valproic Acid Treatment Mediated by Histone Deacetylase Inhibitor Activity in Four Transgenic X. laevis Models of Retinitis Pigmentosa. J Neurosci. 2017;37(4):1039-54.

71. de Vries WN, Binns LT, Fancher KS, Dean J, Moore R, Kemler R, et al. Expression of Cre recombinase in mouse oocytes: a means to study maternal effect genes. Genesis. 2000;26(2):110-2.

72. Lan ZJ, Gu P, Xu X, Jackson KJ, DeMayo FJ, O'Malley BW, et al. GCNF-dependent repression of BMP-15 and GDF-9 mediates gamete regulation of female fertility. EMBO J. 2003;22(16):4070-81.

73. Molday RS, MacKenzie D. Monoclonal antibodies to rhodopsin: characterization, crossreactivity, and application as structural probes. Biochemistry. 1983;22(3):653-60.

74. Preibisch S, Saalfeld S, Tomancak P. Globally optimal stitching of tiled 3D microscopic image acquisitions. Bioinformatics. 2009;25(11):1463-5.

75. Schindelin J, Arganda-Carreras I, Frise E, Kaynig V, Longair M, Pietzsch T, et al. Fiji: an open-source platform for biological-image analysis. Nat Methods. 2012;9(7):676-82 .

76. He F, Nichols RM, Kailasam L, Wensel TG, Agosto MA. Critical Role for Phosphatidylinositol-3 Kinase Vps34/PIK3C3 in ON-Bipolar Cells. Invest Ophthalmol Vis Sci. 2019;60(8):2861-74. 
bioRxiv preprint doi: https://doi.org/10.1101/2021.1011463949; this version posted October 11,2021 . The copyright holder for this preprint

(which was not certified by peer review) is the author/funder, who has granted bioRxiv a license to display the preprint in perpetuity. It is made available under aCC-BY-NC 4.0 International license.

1136 77. Chiang WC, Kroeger H, Sakami S, Messah C, Yasumura D, Matthes MT, et al. Robust 1137 Endoplasmic Reticulum-Associated Degradation of Rhodopsin Precedes Retinal Degeneration. 1138 Molecular Neurobiology. 2015;52(1):679-95.

1139 78. Tawfik A, Smith SB. Increased ER stress as a mechanism of retinal neurovasculopathy 1140 in mice with severe hyperhomocysteinemia. Austin journal of clinical ophthalmology.

1141 2014;1(5):1023-.

1142 